\title{
Turbulent boundary layers and channels at moderate Reynolds numbers
}

\author{
JAVIER JIMÉNEZ $Z^{1,2} \dagger$, SERGIO HOYA $S^{1,3}$, \\ MARK P. SIMENS ${ }^{1}$ AND YOSHINORI MIZUNO \\ ${ }^{1}$ School of Aeronautics, Universidad Politécnica de Madrid. 28040 Madrid. Spain \\ ${ }^{2}$ Center for Turbulence Research, Stanford University, Stanford. CA 94305, USA \\ ${ }^{3} \mathrm{CMT}$ Motores Térmicos, Universidad Politécnica de Valencia, 46022 Valencia, Spain
}

(Recejved 26 October 2009; revised 12 March 2010; accepted 12 March 2010; first published online 2 June 2010)

The behaviour of the velocity and pressure fluctuations in the outer layers of wall-bounded turbulent flows is analysed by comparing a new simulation of the zero-pressure-gradient boundary layer with older simulations of channels. The $99 \%$ boundary-layer thickness is used as a reasonable analogue of the channel half-width. but the two flows are found to be too different for the analogy to be complete. In agreement with previous results, it is found that the fluctuations of the transverse velocities and of the pressure are stronger in the boundary layer, and this is traced to the pressure fluctuations induced in the outer intermittent layer by the differences between the potential and rotational flow regions. The same effect is also shown to be responsible for the stronger wake component of the mean velocity profile in external flows, whose increased energy production is the ultimate reason for the stronger Huctuations. Contrary to some previous results by our group, and by others, the streamwise velocity fluctuations are also found to be higher in boundary layers, although the effect is weaker. Within the limitations of the non-parallel nature of the boundary layer, the wall-parallel seales of all the fluctuations are similar in both the flows, suggesting that the scale-selection mechanism resides just below the intermittent region, $y / \delta=0.3-0.5$. This is also the location of the largest differences in the intensities, although the limited Reynolds number of the boundary-layer simulation $\left(R e_{\theta} \approx 2000\right)$ prevents firm conclusions on the scaling of this location. The statistics of the new boundary layer are available from http://torroja.dmt.upm.es/ftp/blayers/.

\section{Introduction}

This paper analyses the results of a relatively large-scale new direct simulation of a turbulent boundary layer (Simens et al. 2009), with emphasis on the differences between external and internal turbulent Hows.

Turbulent boundary layers have been subjects of interest from the first days of fluid mechanics, especially the canonical case with zero pressure gradient. As a consequence, they were among the first Hows to be simulated (Spalart 1988; Spalart \& Watmuff 1993), but the Reynolds numbers of those simulations have increased more slowly 
than for streamwise-homogeneous flows, such as channels (Kim, Moin \& Moser 1987; del Álamo \& Jiménez 2003; del Álamo et al. 2004; Hoyas \& Jiménez 2006). Part of the reason is that boundary layers are harder to compute, because they are inhomogeneous in at least two directions, but equally important is that they require inflow boundary conditions, which makes their simulations less universal than those of pipes and channels. Even in the relatively straightforward transitional case, the question of how to seed the perturbations has to be considered, and the requirement of simulating the relatively thin initial laminar boundary layer adds greatly to the computational cost. That is why experimental boundary layers are usually tripped, and why some such device is needed in simulations.

Several numerical 'tripping' schemes have been introduced over time, and the Reynolds numbers of the resulting simulations have steadily increased (Alam \& Sandham 2000; Skote \& Henningson 2002; Khujadze \& Oberlack 2004; Ferrante \& Elghobashi 2005; Lee \& Sung 2007), although often using relatively short domains and coarse resolutions. In some of these cases, for example, the zero-pressure-gradient layer is included as an auxiliary to a more complex flow, such as separation in Alam \& Sandham (2000) and Skote \& Henningson (2002), or roughness in Lee \& Sung (2007), and the statistics are not very complete. For this reason, the reference boundary layer simulation was until recently the one by Spalart (1988), with a Reynolds number based on the momentum thickness $\operatorname{Re}_{\theta}=1410$. The simulation on which this paper is based uses the recycling scheme of Lund, Wu \& Squires (1998) to bypass transition, and reaches $R e_{\theta}=2100$. The more recent one by Schlatter et al. (2009), which is comparable to the present one, although at a slightly lower resolution, extends to $R e_{\vartheta}=2500$, but the friction Reynolds numbers of even these newer boundary layers $\left(\delta^{+} \approx 700-800\right)$ are still lower than the intermediate channel simulations mentioned above (e.g. $\delta^{+}=935$ for del Álamo et al. 2004).

Nevertheless, it begins to be possible to compare the properties of simulated boundary layers and channels in the same range of Reynolds numbers, which is the purpose of the present paper. It was found by Jiménez \& Hoyas (2008), after examining a relatively wide range of experiments and simulations, that internal and external wall-bounded turbulent flows are noticeably different, especially in the behaviour of the two transverse velocity components and of the pressure. More recently, Buschmann et al. (2009) extended that analysis to a larger data set, and confirmed that the outer regions of external flows contain structures, involving the transverse velocities, which are not present, or are much weaker, in internal ones. The nature of those structures is unclear, and Buschmann et al. (2009) raised the possibility that a different mechanism may be responsible for the excess of each of the two velocities. Part of the problem is that, for the reasons just mentioned, most of the data for external flows have been up to now experimental, restricted to a fairly small set of variables. This paper can be considered, in some sense, as a continuation of those two previous ones. Our goal is to use the more complete database from the new simulation to study the reasons for the observed differences, and in particular to elucidate the effect of large-scale intermittency, which is the most obvious phenomenon present in external Hows, but not in internal ones.

The structure of the paper is as follows. The basic code and simulation are introduced in $\$ 2$, and the results are briefly described in $\$ 3$. The eflect of intermittency is discussed in $\$ 4$, followed in $\$ 5$ by the analysis of the related information provided by the spectra. The paper then summarizes and concludes. Jiménez et al. (2009) is a preliminary version of the present discussion. The statistics of the new simulation are available from http://torroja.dmt.upm.es/ftp/blayers/. 


$\begin{array}{cccccc}R e_{\theta} & \left(L_{x}, L_{y}, L_{z}\right) / \theta & \Delta x^{+}, \Delta y^{+}, \Delta z^{+} & \Delta y / \eta & N_{x}, N_{y}, N_{z} & T u_{z} / \delta_{99} \\ 620-2140 & 535 \times 29 \times 88 & 6.1 \times 0.30 \times 4.1 & 1.4 & 6145 \times 360 \times 1536 & 21\end{array}$

TABLE 1. Parameters of the boundary-layer simulation. $L_{x}, L_{y}$ and $L_{z}$ are the box dimensions along the three axes. $N_{x}, N_{y}$ and $N_{z}$ are the corresponding grid sizes, expressed for $z$ in terms of collocation points, and the various $\Delta$ valnes are the resolutions, given at their coarsest points. The Kolmogorov length $\eta$ is computed from the local energy dissipation. The coarsest resolution along $x$ and $z$ in terms of $\eta$ is found at the wall, where $\eta^{+} \approx 1.5$. The resolution given for $y$ is reached at $y \approx \delta_{99} / 2$, where $\eta^{+} \approx 3$. The time nsed for the statistics is $T$, after discarding transients. Reference quantities used for normalization are taken midway into the simulation box.

\section{The numerical simulation}

The boundary layer is simulated in a parallelepiped over a smooth no-slip wall, spatially periodic spanwise, but with non-periodic inflow and outflow in the streamwise direction. The numerical code uses a relatively classical fractional-step method (Kim \& Moin 1985; Perot 1993) to solve the incompressible Navier-Stokes equations expressed in primitive variables. It is discussed in detail in Simens (2008) and Simens et al. (2009), which also contain examples of applications to other problems. The simulation parameters are summarized in table 1.

The velocity components in the streamwise $(x)$, wall-normal $(y)$ and spanwise $(z)$ directions are $u, v$ and $w$, respectively, and the kinematic pressure is $p$. Uppercase symbols refer to mean quantities, and lower-case symbols are reserved for fluctuations. Wall-scaled variables are defined in terms of the local friction velocity $u_{r}$ and of the molecular viscosity $\nu$, and are denoted by a superscript ' + '. Other quantities used throughout the paper are the free-stream velocity $U_{\alpha,}$, the momentum and displacement thicknesses $\theta$ and $\delta^{*}$, and the $99 \%$ boundary-layer thickness $\delta_{99}$. The subscripts ' 0 ' and ' $e$ ' denote the inhlow and out llow sections. Primed quantities, such as $u^{\prime}$, refer to root-mean-squared Huctuation intensities.

The no-slip wall is the bottom $(x-z)$ plane, and the velocities at the outflow are estimated by a convective boundary condition, with small corrections to enforce global mass conservation (Simens et al. 2009). Time stepping is by the semi-implicit three-step Runge-Kutta scheme of Spalart, Moser \& Rogers (1991). The nonlinear and wall-parallel viscous terms are treated explicitly, with the only implicit part being the linear viscous terms in the $y$ direction. The time step is adjusted to a constant $C F L=0.6$, to preserve time accuracy. The convective and viscous terms in the $x$ and $y$ directions are computed using staggered three-point compact finite differences (Nagarajan, Lele \& Ferziger 2003), while the velocity and pressure are expanded in Fourier series along $z$. No staggering is used in that direction, and the computation of the nonlinear terms is pseudospectral, using the $2 / 3$ rule to prevent aliasing.

The turbulent inflow is generated by the recycling scheme of Lund et al. (1998), in which the velocities from a reference downstream plane, $x_{\text {ref }}$, are used to synthesize the incoming turbulence. This was the source of several numerical difficulties, described in detail by Simens et al. (2009). The result is that the initial $300 \theta_{0}-400 \theta_{0} \approx 35 \delta_{99,0}-50 \delta_{99,0}$ of the simulation domain have to be discarded. For example, the maxima of the fluctuation intensities only reach what appear to be their asymptotic gradual growths after that length, and a similar conclusion can be drawn from the decay of the space-time velocity correlations (Simens et al. 2009). The reason for this long adaptation length is not solely numerical. The turnover time of the largest eddies is 
of the order of $\delta_{99} / u_{T}$, during which time the eddies are advected over a distance $U_{\infty 0} \delta_{9 g} / u_{\tau} \approx 22 \delta_{9 g} \approx 200 \theta$. The inflow length mentioned above is therefore about two eddy turnovers, comparable to the initial simulation time routinely discarded from turbulent channel simulations. Of course, at least an initial washout time also has to be discarded from the boundary-layer simulation. Another necessary precaution is that the reference plane has to be located well beyond the end of the inflow region to avoid spurious feedbacks (Nikitin 2007; Simens et al. 2009). In our case it is located at $x_{r e f} / \theta_{0}=850\left(R e_{\theta}=U_{\infty} \theta / v=1710\right)$. The simulation was initialized from a filtered field from Spalart (1988), extended gradually downstream and did not require especial precautions to maintain the turbulent state.

The average streamwise pressure gradient is controlled by applying a constant uniform suction at the upper boundary, which is otherwise stress-free. The transpiration velocity is estimated from the known experimental growth of the displacement thickness in that range of Reynolds numbers. This keeps the acceleration coefficient $\beta=\delta^{*} U_{i c}^{+} \partial_{x} U_{x_{*}}^{+} \approx 2 \times 10^{-4}$, which is reasonably small. The pressure gradient increases sharply to $\beta \approx 5 \times 10^{-3}$ within the last $5 \%$ of the numerical domain, corresponding to the last 1.5 boundary-layer thicknesses. That is clearly due to the effect of the outflow, which uses no numerical sponge in this particular simulation, and that region is discarded from the results. Together with the inflow length mentioned above, the discarded region amounts to approximately one-third of the simulation box, and limits the useful range of Reynolds numbers to about $R e_{\theta} \approx 1100-2050$. from the one given in table 1 .

The intensity of the free-stream velocity fluctuations is controlled by the ratio between the height of the computational box and the boundary-layer thickness at the exit, $\delta_{99}$, and remains almost constant with $x$. The free-stream intensity of the present simulation is $u^{\prime} \approx 2.5 \times 10^{-3} U_{x,}$, and is associated with large-scale vorticity fluctuations of the order of $2 \times 10^{-3} U_{\infty} / \delta_{99 e}$, introduced at the inflow by the sloshing created by the interaction of the boundary layer with the exit.

\section{Basic statistics}

Figure 1(a) shows the development of the friction coefficient of the simulation, $c_{f}=2 / U_{x^{\prime}}^{+2}$, as a function of $R e_{\theta}$, compared with other simulations and experiments in roughly the same range of Reynolds numbers. The experiments of Erm \& Joubert (1991) are especially useful to estimate the location beyond which the simulation can be considered as roughly independent of the inflow condition, because they were repeated with three different tripping devices, plotted in figure 1 with different symbols. Their conclusion was that the effect of the trip survives up to $R e_{\theta} \approx 1500$, and only becomes small beyond that limit. It is seen in figure $1(a)$ that the same is true for our results, which initially diverge widely from the experiments, but eventually settle into excellent agreement with them at about the same location at which the experimental scatter begins to decrease. Figure 1(a) also includes the limits of the useful region, which were determined in Simens et al. (2009), in part by analogy with the tripping experiments, and in part from the arguments outlined in the previous section. The reader is referred to that work for details, but note that the tripping results suggest that the first half of that 'useful' range may retain some memory of the inflow.

The figure also displays the segments used to average the statistics of the three reference sections used later in the paper, listed in table 2 . Each of them is about $2 \delta_{99}$ long. The averaging reduces the statistical noise and does not induce large errors. 

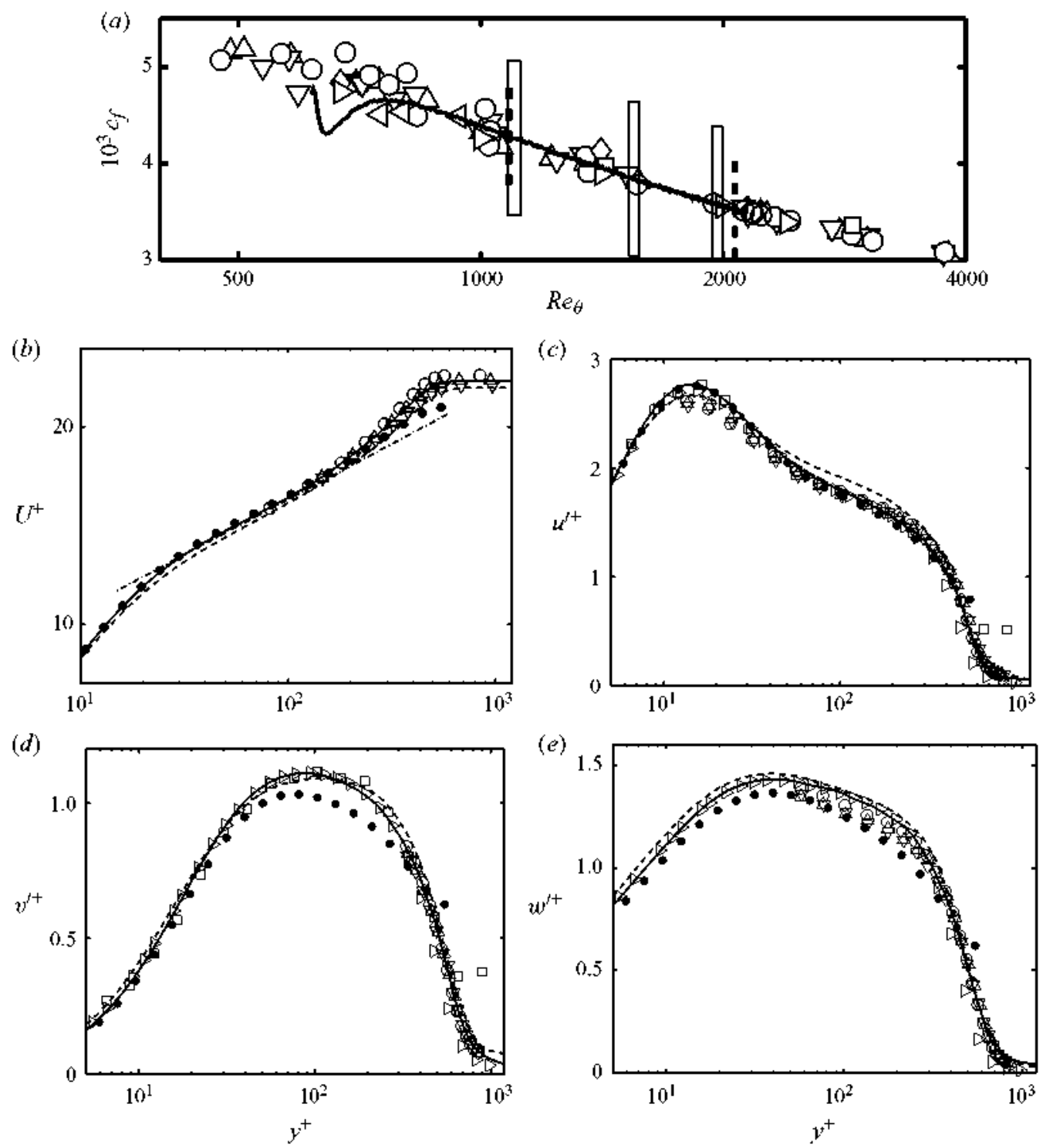

FIGURE 1. (a) Friction coefficient of bonndary layer versus Reynolds nnmber. Symbols are experiments by Erm \& Jonbert (1991), tripped by $\triangle$, wire; $\nabla$, grit;, , pins; ', simnlations by Spalart (1988); $\triangleright$, simulations by Schlatter et al. (2009); $\triangleleft$, simulations by Wu \& Moin (2009); $\square$, experiments by de Graaff \& Eaton (2000); - present simulation. The dashed vertical lines are the limits of the nseful range, as discussed in the text, and the three narrow rectangles are the averaging ranges for sections BLS1-BLS3 in table 2. (b) Mean streamwise velocity. $\longrightarrow$, Present simulation at $R e_{\theta}=1350 ;----$, Spalart (1988), $R e_{\theta}=1410 ; \bullet$, numerical channel C 550; open symbols are as in $(a)$, with $R e_{\theta} \approx 1350 .--, \log \left(v^{+}\right) / 0.41+5.1 .(c-e)$ Root-mean-squared velocity Huctuations. Symbols as in $(b)$, with $R e_{\theta} \approx 1550$ for Erm \& Joubert (1991), $R e_{\theta}=1450$ for the present simulation, $R e_{\theta}=1430$ for de Graafl \& Eaton (2000), and $R e_{\theta}=1410$ for Schlatter et al. (2009).

The most serious one is due to the variation of the boundary-layer thickness over the averaging segment, which is slow in the zero-pressure-gradient case. It stays below $\pm 1.5 \%$ for the segments used here. Since the relative averaging error is only due to 


\begin{tabular}{lcccccccccc}
\hline & $\delta^{+}$ & $L_{x} / \delta$ & $L_{y} / \delta$ & $L_{z} / \delta$ & $x / \theta_{0}$ & $R e_{\theta}$ & $\theta / \theta_{0}$ & $\delta^{*} / \theta$ & $\delta 99 / \theta$ & $U_{i \circ}^{+}$ \\
BLS1 & 444 & 80.2 & 4.3 & 13.2 & 355 & 1100 & 1.78 & 1.435 & 8.76 & 21.7 \\
BLS2 & 580 & 59.0 & 3.2 & 9.7 & 710 & 1550 & 2.51 & 1.421 & 8.53 & 22.8 \\
BLS3 & 692 & 47.4 & 2.6 & 7.8 & 1070 & 1970 & 3.20 & 1.415 & 8.31 & 23.6 \\
C550 & 550 & $8 \pi$ & 2 & $4 \pi$ & (del Álamo \& Jiménez 2003) & & \\
C950 & 935 & $8 \pi$ & 2 & $3 \pi$ & (del Álamo et al. 2004) &
\end{tabular}

TABLE 2. Parameters of the numerical data sets nsed in the paper. BLS1 to BLS3 are three streamwise stations from the bonndary-layer simulation. Each station is averaged over 150-250 neighbouring points, corresponding locally to $2 \delta_{99}$. The momentum thickness at the inflow is $\theta_{0}$. C550 and C950 are older numerical channels used as comparisons. The friction Reynolds nnmber $\delta^{+}$is based on the half-width for the channel, and on $\delta_{99}$ for the bonndary layer. More data about the two channels are fonnd in the original pnblications in the table.

the quadratic terms of the downstream evolution of the statistics, it should be smaller than about $10^{-3}$.

Figures $1(b)$ and $1(c)$ present mean velocity profiles and streamwise fluctuation intensities near the centre of the computational domain. They also include the closest available experimental Reynolds numbers from Erm \& Joubert (1991), and from simulations at roughly similar Reynolds numbers. The agreement is excellent, especially with the experiments, and with the simulations of Schlatter et al. (2009) below $y / \delta_{99} \approx 0.6$. The minor discrepancies between the intensities of Spalart (1988), both with the present results and with the experiments, cannot be attributed to the Reynolds number difference, and are presumably a consequence of the mean-flow expansion used by him to approximate the flow, although the recent note by Spalart, Coleman \& Johnstone (2009) suggests that their resolution was also slightly too coarse. The slightly lower intensities of Erm \& Joubert (1991) near the wall are also probably due to a minor under-resolution of the experiments in that region, since the length of their hot wire was approximately 20 wall units, and their innermost data points were very close to the intensity maximum. Our simulation agrees much better with the intensities from de Graafl \& Eaton (2000), which are very well resolved near the wall. Note that the statistics used in this figure are not averaged over a range of $x$, and are chosen in each case to match the available experimental and simulation data as closely as possible.

Figures $1(b)$ and $1(c)$ also include data from the channel C550, and the agreement is reasonable, except for the outer-layer 'wake' deviation of the mean velocity profile with respect to the logarithmic law, which is well known to be weaker in channels. A somewhat smaller discrepancy of the streamwise fluctuations in the wake region is masked in figure $\mathbf{1}(\mathrm{c})$ by the logarithmic scaling of the abscissae, and will be discussed later in the context of a more complete comparison of the two Hows. It should be noted in that respect that it is not immediately obvious what thickness should be used to normalize the diflerent profiles, or to compute the Reynolds numbers. Jiménez \& Hoyas (2008) concluded that a reasonable choice was to use $\delta_{99}$ for boundary layers, and the half-width for channels. We retain that convention here, and will loosely refer to both quantities as $\delta$. For example, for the boundary layer and channel simulations in figure 1 , the two Reynolds numbers are $\delta_{99}^{+} \approx 580$ and $\delta^{+}=547$.

The agreement in figure $1(b, c)$ does not hold for the transverse velocities intensities in figure $\mathbf{1}(d, e)$, or for the pressure fluctuations in figure 2 , all of which are stronger in the boundary layers than in the channels. This was already noted by Jiménez \& 

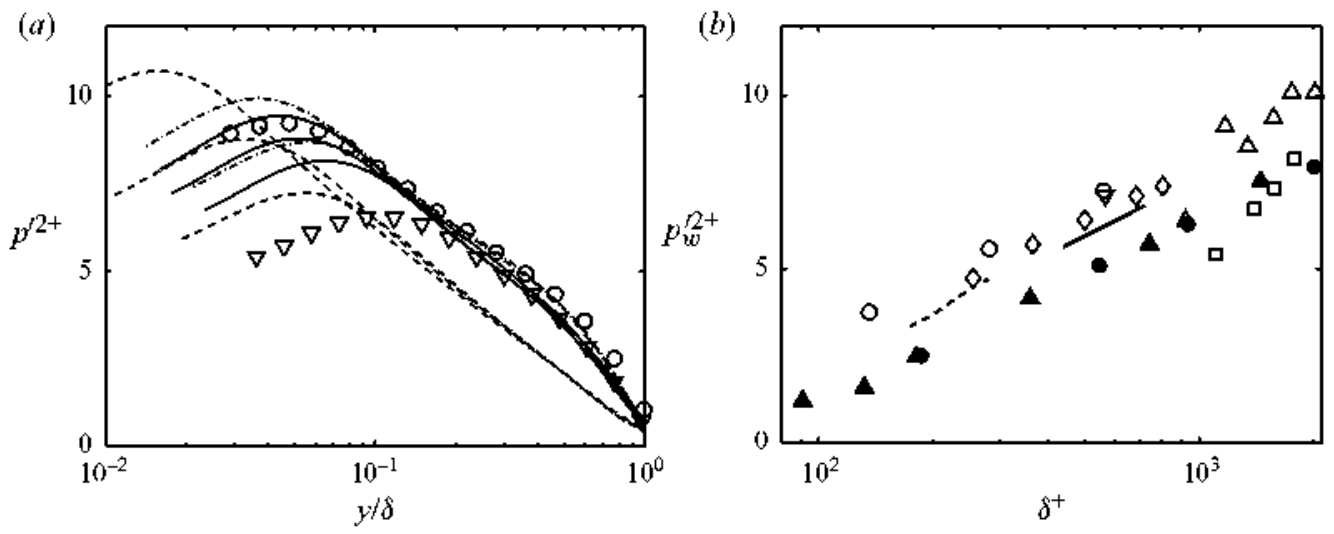

FIgure 2. (a) Profiles of the pressnre fluctuation intensities. ----, Nnmerical channels from table 2 and Hoyas \& Jiménez (2006), $\delta^{+}=550-2003 ;-$, present bonndary layer, $\delta^{+}=445-690 ; 0$, numerical boundary layer, $\delta^{+}=560$ (Spalart 1988); $\nabla$, numerical boundary layer, $\delta^{+}=280$ (Skote, Haritonides \& Henningson 2002); - - - , numerical boundary layer, $\delta^{+}=500$ and 800 (Schlatter et al 2009 ). (b) Pressnre Huctuation intensities at the wall. Nnmerical channels: $\bullet$, from table 2; $\boldsymbol{\Delta}$, (Hn, Morley \& Sandham 2006). Numerical bonndary layers: - , present; ----, (Skote et al. 2002); o, (Spalart 1988). \%, (Schlatter et al. 2009). Experimental boundary layers: $\nabla$ (Schewe 1983); $\Delta$ (Farabee \& Casarella 1991); $\square$ (Tsuji et al. $2007)$.

Hoyas (2008) on the basis of incomplete, and generally noisy, experimental data, and could perhaps be interpreted as meaning that the reference length for boundary layers should be taken larger than $\delta_{9 g}$. Since most intensities grow slowly with the Reynolds number in the range of the simulations, this would improve the agreement between the two Hows. However, the thickness needed to match the transverse velocities and pressures near the wall would be $\delta \approx 1.7 \delta_{99}$, which is rather large, and which fails to match the profiles above the bufler layer. Using $1.7 \delta_{99}$ as a reference length for the boundary layers would also spoil the agreement in figure $1(\mathrm{c})$. There is indeed no reason to suppose that the same length scale should work for all the variables, or across the whole flow. The boundary-layer thickness is associated with the outer flow, and the most reasonable interpretation of the results in figure 1 is that the outer parts of boundary layers and channels are intrinsically different.

The pressure fluctuations, which are difficult to obtain from experiments, deserve some discussion. The profiles in figure $2(a)$, which come from simulations, fall into two distinct families, each of which collapses in wall units with very little noise. There are no experimental pressure profiles at comparable Reynolds numbers. The pressure fluctuations at the wall are represented in figure $2(b)$, and include both experiments and simulations. The seatter of the numerics is again small, and even that of the experiments would be reasonable, except for the single experiment by Tsuji et al. (2007), which differs from most other experiments in that range. It is difficult to give a reason for that discrepancy without access to the full experimental details, but private consultations with the leading author of that paper suggest that those data, which correspond to the lowest Reynolds number range of their experiment, may not have been sufficiently corrected for the presence of background acoustic noise. If those data are set apart, the separation of figure $2(b)$ into internal and external families is clear cut. 

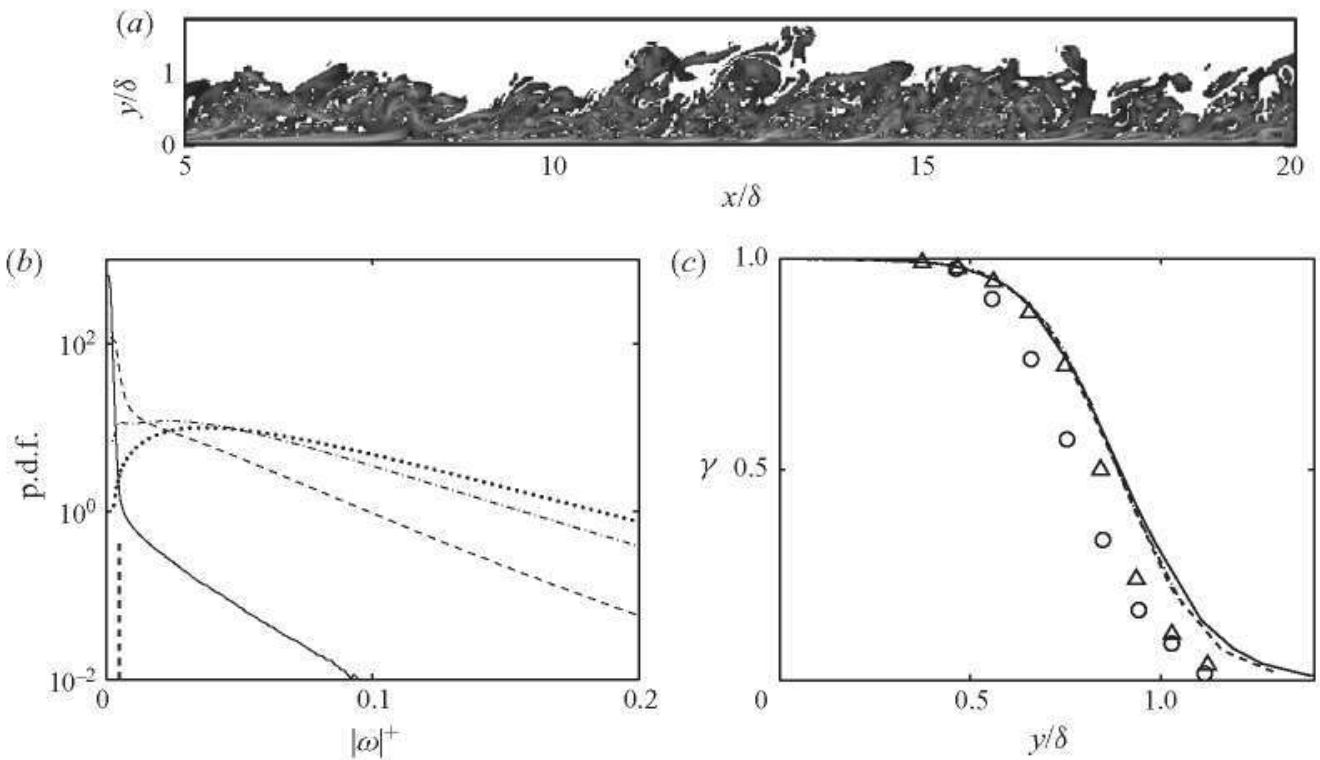

Figure 3. (a) Typical section of $\left|\omega^{\prime}\right|$ in the boundary layer, showing intermittent potential flow deep into the vortical region. $R e_{\theta} \approx 700-900$. (b) Probability density functions of the vorticity magnitude in section BLS2, showing the development away from the wall of the irrotational delta at $|\omega| \approx 0 . \cdots \cdots \cdots, y / \delta_{99}=0.44 ;-.-, 0.59 ;-\cdots, 0.88 ;-, 1.31$. The dashed vertical line is the limit used to define irrotational flow, slightly larger than a single histogram bin. (c) Intermittency factor. - - BLS1 in the present simulation;----, BLS2; - - - BLS3; 0 , from experimental velocity measurements at $\operatorname{Re}_{\theta}=3000$ (Kovasznay, Kibens \& Blackwelder 1970); $\triangle$, from temperature measurements at $R e_{\theta}=1100-4800$ (Murlis, Tsai \& Bradshaw 1982).

\section{Intermittency}

The most obvious difference between the two flows is that the outer part of boundary layers is intermittent, whereas that of channels is not. Intermittency is used here in the original sense of the large-scale coexistence of irrotational and rotational fluid near the edge of the turbulent region (Corrsin \& Kistler 1955). In particular, we will define the intermittency coefficient $\gamma$ as the fraction of time for which the flow is rotational at a given location. This quantity was widely used in the early days of boundarylayer research, although mostly as a means of studying the turbulent-irrotational interface, and continues to be used extensively in turbulence modelling, because the irrotational fraction strongly influences the flow behaviour (Pope 2000). The dynamics of the interface continues to be the subject of current research (Westerweel et al. 2009), mostly in free shear flows, but we will restrict ourselves here to the effect of intermittency on the behaviour of the energy-containing flow scales.

The measurement of intermittency was difficult in early laboratory experiments because it required the arbitrary estimation, from one-dimensional velocity signals, of whether the flow was irregular enough to be considered turbulent (Corrsin \& Kistler 1955; Kovasznay et al. 1970), or the use of surrogates such as the transport of passive scalars (Fiedler \& Head 1966; Murlis et al. 1982). The definition can be made more precise in simulations, because the vorticity magnitude $|\omega|$ can be computed, and irrotational flow can be characterized as where the vorticity vanishes (Bisset, Hunt \& Rogers 2002). An example is figure 3(a), which shows a typical instantaneous vorticity field in a short section of the boundary-layer simulation. The open irrotational regions 
extend well within the darker vortical layer. If the probability density function (p.d.f.) of $|\omega|$ is computed for a given wall distance, as in figure $3(b)$, those regions appear as a delta function at $|\omega|=0$. The probability contained in those deltas is $1-\gamma$.

The result is displayed in figure 3(c), compared with older experimental values. The agreement is excellent, considering the differences in Reynolds numbers and in identification techniques, and shows that the irrotational fraction begins to be substantial above $y \approx \delta_{99} / 2$. It dominates the flow for $y \geq \delta_{99}$, but some vortical fuid remains even for $y / \delta_{99} \approx 1.25$. The somewhat higher intermittency values of the simulation with respect to the experiments are probably real, because our identification method, which does not depend on a threshold or on the properties of the signal over an extended segment, identifies small vortical structures that would be neglected by the older schemes. Note that the intermittency profiles of our three boundary-layer sections, which differ in Reynolds number by a factor of about 1.5, fall on top of each other within the measurement accuracy, suggesting that the outer part of the boundary layer is relatively independent of the Reynolds number, and therefore also probably relatively independent of the near-wall region.

The effect of intermittency can be studied by means of two-dimensional p.d.f.s of the different variables with $|\omega|$, which allows the computation of statistics conditioned to potential and rotational fluid. An example is given in figure $4(a)$ which shows that the mean streamwise velocity is higher in the potential region than in the rotational one. This makes sense, because the potential flow has to come from the free stream and, in the absence of turbulence, can only be slowed by large-scale pressure gradients. Although not shown in the figure, the irrotational regions in the intermittent layer have negative mean wall-normal velocities, while the rotational ones are weaker outflows (Kovasznay et al. 1970; Hedley \& Keffer 1974). By itself, this would explain why the streamwise velocity is higher in the downdrafts, but the effect is stronger than that, as can be seen by comparing figure $4(a)$ with figure $4(b)$, which shows the same quantity conditioned to positive and negative wall-normal velocity, without reference to the vorticity.

Fiedler \& Head (1966) and Kovasznay et al. (1970) had already shown that the velocity of the potential fluid in boundary layers is much closer to the free stream than the average, and the same was shown in two-dimensional free mixing layers by Wygnanski \& Fiedler (1970). Our results agree broadly with those experiments. For example, Kovasznay et al. (1970) found $U_{\text {pot }} / U_{\text {rot }}=1.033$ at $y=0.8 \delta_{99}$, while our corresponding value is 1.035 .

It has often been proposed that the reason why the high-speed 'wake' of the outer layer of the mean velocity profile is much stronger in boundary layers than in internal flows has to do with the intermittent behaviour of the former (see e.g. Murlis et al. 1982). The high velocity of the irrotational regions provides a plausible mechanism. The very different dynamics of the rotational and potential flow in the intermittent layer is shown by the conditional Reynolds stress in figure $4(\mathrm{c})$. Normally, one would expect the tangential Reynolds stress, $-\langle a v\rangle$, to be positive everywhere in the How, but that is not the case here. While the potential fluid is strongly slowed as it enters the layer, the averaged Reynolds stress in the rotational part is contrary to the overall mean value, and also acts to slow the rotational fluid as it rises. The strong contribution of the irrotational part appears to contradict the common wisdom that potential How cannot support Reynolds stresses, but is due to our choice of the overall average velocity as the reference for the fluctuations. The irrotational flow entering the layer brings with it the higher momentum of the free stream, which appears as a Reynolds stress with respect to the mean. When the fluctuations are defined 

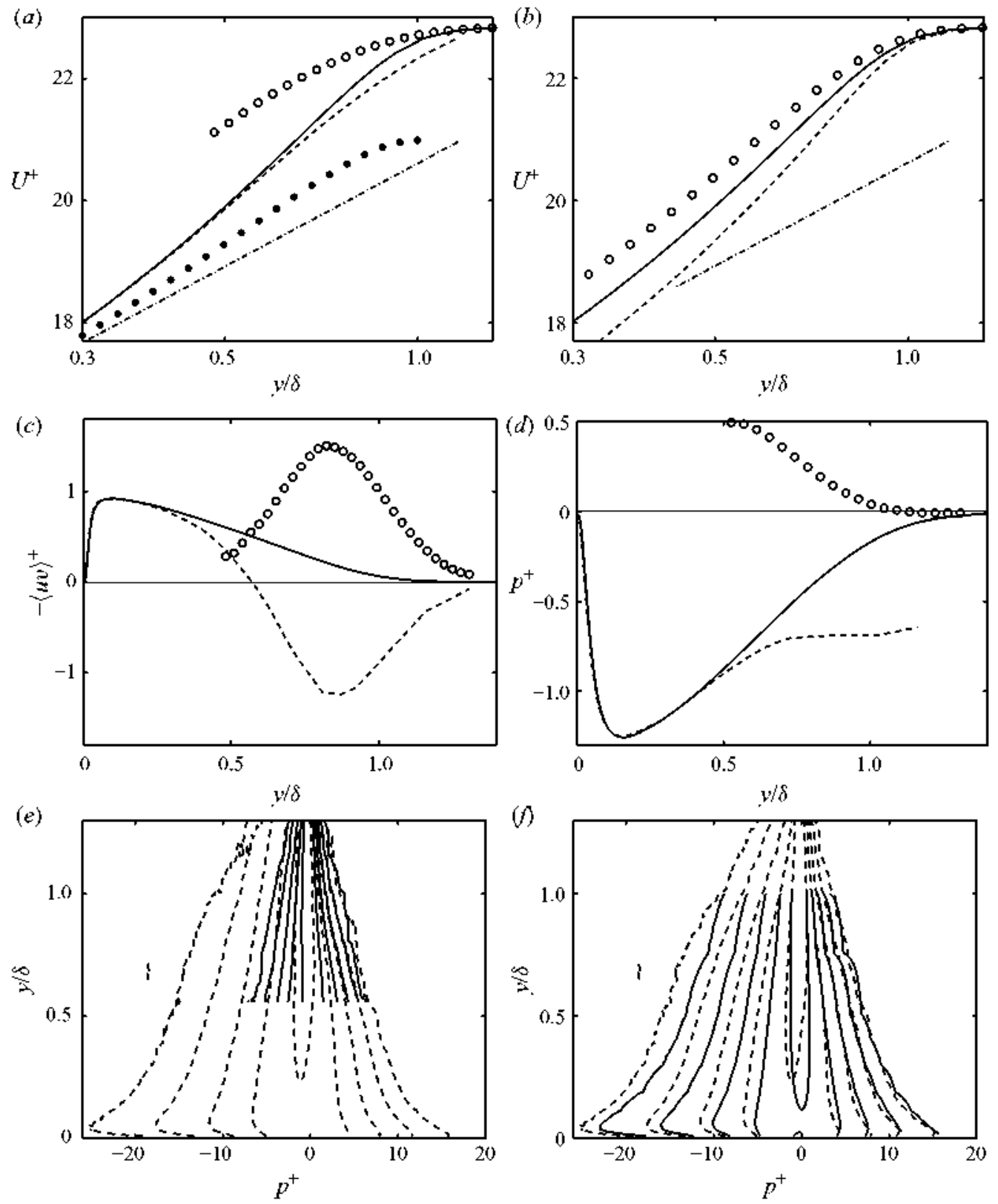

Figure 4. Conditional statistics from station BLS2 of the present simulation. - Unconditional average. $(a, b)$ Mean streamwise velocity; the chain-dotted line is $\log \left(y^{+}\right) / 0.41+5.1 ; \bullet, C 550$ channel. Note that the horizontal axes are logarithmic (c) Reynolds stress $-\langle u v\rangle,(d)$ Mean pressure. In $(a, c, d)$, irrotational mean:----, rotational mean. In (b) $0, v \leqslant 0 ;---, v>0$. (e) Conditional one-dimensional p.d.f. of the pressure for the boundary layer at BLS2, as a function of wall distance. - - Irrotational flnid; ----, rotational. $(f)$ Unconditional one-dimensional p.d.f. of the pressure, as a function of wall distance.--BLS2; $\longrightarrow$, C550. The isolines in (e) and $(f)$ are spaced by factors of 10 , down from $10^{-1}$. 
with respect to the mean velocity conditioned to each kind of Huid, the irrotational Reynolds stress is very close to zero, as expected, while the stress in the vortical flow is somewhat higher than the overall mean to compensate for its smaller time fraction. This agrees with older measurements by Hedley \& Kefler (1974), but neglects the large-scale momentum transfer of the irrotational inrushes.

In the absence of intrinsic Reynolds stresses to slow the motion of the irrotational regions, the homogenization of the velocities can only take place through the pressure. In the same way, the negative Reynolds stresses of the rotational part imply that the vortical fluid is being accelerated by some mechanism other than advective momentum transfer. How this takes place is shown in figure $4(d)$. The pressure in the potential regions is higher than in the free stream, while that in the rotational ones is lower. One could think of the incoming fast potential How as pushing into the slower rotational one to its front, while sucking the one behind. It is interesting that the pressure fluctuation profiles of the boundary layers in figure 2 are roughly parallel to those of the channels, and that their oflset is mostly due to the faster rise of the fluctuations across the intermittent part of the boundary layers. Pressure is a global quantity, especially when it is generated by spatially extended sources (Kim 1989; Jiménez \& Hoyas 2008), and it is tempting to identify the extra pressure fluctuations as those coming from the intermittent layer.

In fact, the origin of those fluctuations can be traced in some detail. Figure 4(e) shows the individual p.d.f.s of the pressure for the irrotational and rotational Huids, as a function of wall distance, and it is clear that the reason for the lower mean pressure in the rotational part is the presence of a low-pressure tail that is absent from the potential Huid. Those negative tails are usually attributed to the low-pressure regions in the cores of the vortices. The pressure p.d.f.s in the potential regions are roughly symmetric, lacking vorticity, and the positive tails of the two Huids, traditionally associated with strain-dominated regions, are almost identical. The same is true for the comparison of the channel with the boundary layer, which is presented in figure $4(f)$. The positive tails are essentially equal, but the rotational tail is stronger in the boundary layer, resulting in larger overall pressure fluctuations.

Note that the decomposition in figure $4(c)$ is not exactly equivalent to the classical quadrant analysis of the Reynolds stresses. The irrotational inrushes are predominantly fourth-quadrant (Q4) sweeps $(u>0, v<0)$, but they are not the only sweeps in the How, in the same way that the turbulent eddies contain both normal Q2 ejections $(u<0, v>0)$ and the Q1 outgoing interactions that eventually result in the overall 'counter-gradient' contributions to the Reynolds stress in figure $\mathbf{4}(\mathrm{c})$. The classical quadrant decomposition of the Reynolds stresses is given in figure 5(a), both for the boundary layer and for a channel at a somewhat higher Reynolds number. In both cases, the contribution of the ejections is larger than that of the sweeps, presumably reflecting the stronger fluctuations near the wall. The imbalance increases away from the wall, but the behaviour is different in the channel and in the boundary layer. In the former, the contribution of the two dominant quadrants, Q2 and Q4, increases near the centreline, and is compensated by a parallel increase of the two counter-gradient quadrants Q1 and Q3 (see e.g. Wallace, Eckelmann \& Brodkey 1972). Most of this increase is simply due to taking fractions with respect to a total stress that vanishes at the centreline, while the contributions of the individual quadrants do not. Some of the structures from one wall cross the centreline into the other half of the channel, and are aliased into a different quadrant. Thus, a Q2 ejection that crosses the centreline masquerades as a Q3 sweep in the other side of the channel. At the centreline itself there is no way to distinguish between Q1 and Q4, or between 

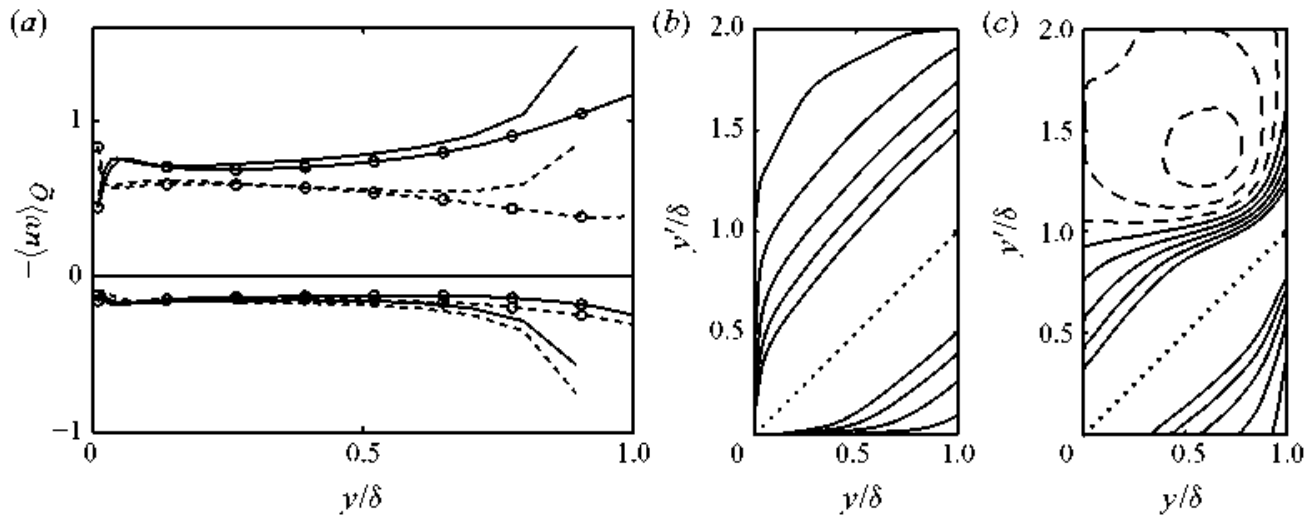

FIGURE 5. (a) Fractional quadrant contribution to the Reynolds stress $-\langle u v\rangle$. Positive values: $\longrightarrow$, Q2 ejectious:----, Q4 sweeps. Negative values:-,- Q1 ejections:----, Q3 sweeps. Lines without symbols are the C950 chanuel, those with circles are the BLS2 boundary layer. $(b-c)$ Correlations $C\left(y, y^{\prime}\right)$ of the velocity fluctuations for $\lambda_{x} / \delta>3$ and $\lambda_{z} / \delta>1.5$ in the $C 950$ channel. Isolines are $-0.2(0.1) 0.5$, and solid ones are positive. The dotted line is $y=y^{\prime}$, where the correlation is unity. (b) Wall-normal velocity. (c) Streamwise velocity.

Q2 and Q3 (Kim et al. 1987). That some structures cross deeply into the opposite half of the channel is shown in figure $5(b)$, which displays the $y$-correlation of the wall-normal velocity,

$$
C_{v v}\left(y, y^{\prime}\right)=\frac{\left\langle v(y) v\left(y^{\prime}\right)\right\rangle}{\langle v(y) v(y)\rangle}
$$

computed for Huctuations that have been filtered to wavelengths larger than $\left(\lambda_{x}, \lambda_{z}\right)=(3,1.5) \delta$. These were the dimensions identified by del Alamo et al. (2004) and del Álamo et al. (2006) for the large-scale $v$-structures in the flow. The correlation crosses the centreline, and it is clear that the large $v$-structures retain their coherence at least across the central $50 \%$ of the channel height. Moreover, although not shown in the figure, the eflect becomes stronger with the Reynolds number, at least between the two channels used in this paper, C550 and C950. On the other hand, it is restricted to the largest scales, and disappears for wavelengths smaller than about $\delta$. The correlation of the streamwise velocity also crosses the centreline (figure $5 \mathrm{c}$ ), but in that case it is antisymmetric. A slow large-scale streak in one side of the channel tends to correspond to a fast one in the other side, no doubt to preserve continuity.

The quadrant structure of the boundary layer in figure $5(a)$ is very similar to that of the channel up to $y / \delta=0.6$, but the two diverge in the intermittent layer. None of the effects in the preceding paragraph are present in the boundary layer, and there is very little growth of the contributions of the two counter-gradient quadrants. On the other hand the contribution of the ejections keeps growing at the expense of the sweeps. While the ratio Q2/Q4 stays in the range 1.5-2 for the channel, in the boundary layer it reaches more than 3 at $y \approx \delta$. This agrees with the decreased efficiency of the irrotational sweeps in the intermittent layer, and suggests that most of the counter-gradient stresses in the turbulent fluid discussed in connection with figure $\mathbf{4}$ are not due to anomalous ejections, but to a lack of high-velocity turbulent fluid to feed sweeps. Although not included in figure 5(a), for clarity of presentation, Nakagawa \& Nezu (1977) presented the quadrant analysis of an open half-channel at similar Reynolds number. That How is not intermittent, and its behaviour is 

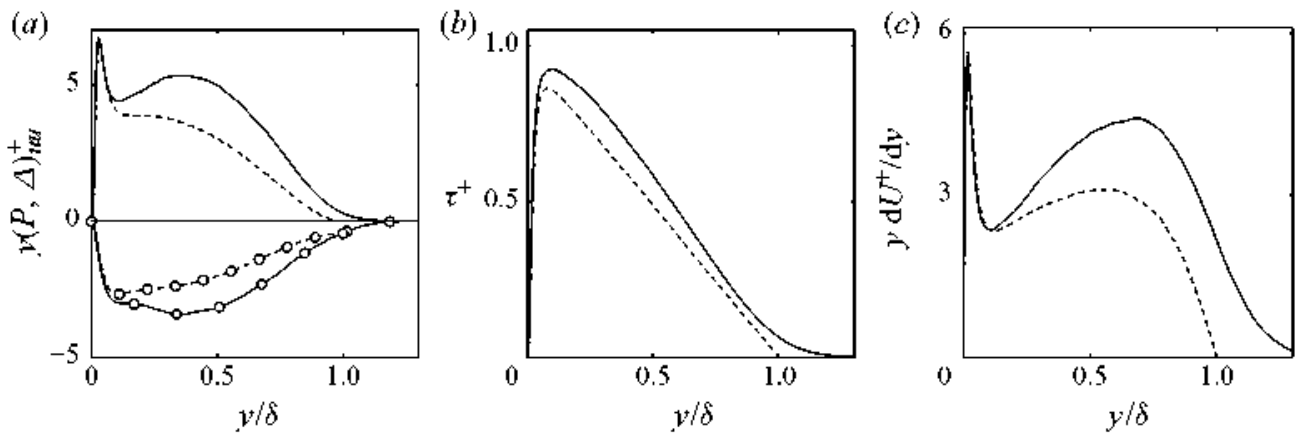

FIGURE 6. Energy bndgets for the streamwise velocity Huctnations. —. Present bonndary layer, BLS2;----, channel C550. (a) Lines without symbols are the production, $\tau \partial_{y} U$, and those with symbols are the pressure-redistribution term towards the two other velocity components. Note that the cnrves are pre-mnltiplied by $y$, to emphasize the outer layers. (b) Reynolds shear stress. (c) Pre-multiplied mean velocity gradient.

intermediate between the channel and the boundary layer, but much closer to the former. In particular, it presents none of the loss of efficiency of the sweeps that we have attributed to intermittency in the boundary layer.

Note that the observation of the differences between the quadrant distribution of boundary layers and channels had already led Antonia et al. (1992) to remark that the interaction between the two channel halves could not be restricted to the neighbourhood of the channel centreline. They attributed that interaction to their low Reynolds number, but the present results suggest that it is a more general property of turbulent channels.

\subsection{Energy balances}

The stronger negative tail shown in figure $4(e, f)$ for the pressure fluctuations in the boundary layer suggests that the vorticity fluctuations should also be stronger, which in turns implies a stronger dissipation and a stronger energy production. Both things turn out to be true.

Figure 6 compares the energy balances of the boundary layer with those given by Hoyas \& Jiménez (2008) for the C550 channel. Figure 6(a) shows the production of the streamwise velocity fluctuations $P_{u t}=\tau \partial_{y} U$, where $\tau=-\langle u v\rangle$ is the Reynolds shear stress. This is, of course, the full energy production, part of which gets redistributed to the transverse velocities by the pressure term $\Delta_{u t}=\left\langle u \partial_{x} p\right\rangle$, which is also given in the figure. Note that the energy budgets have been pre-multiplied by $y$ to emphasize their outer layers. The dominant terms of the energy budgets decay as $1 / y$ above the buffer layer, but their integrated eflect remains important, because $1 / y$ is not integrable for large $y$, and the bufler region is a negligible part of the boundary-layer thickness at large Reynolds numbers.

It is clear that both the production and the pressure term are larger in the boundary layer than in the channel, which helps to explain why the pressure and the transverse velocities are also stronger. Since pressure enforces continuity, it is not surprising that a by-product of its role in homogenizing the differences between the streamwise velocities of the turbulent and potential regions should be to enhance the transverse velocity fluctuations.

The two factors in the energy production are shown independently in figures $6(b)$ and $6(c)$. They show that the main reason for the larger production in the boundary layers is its steeper velocity gradient, emphasizing again the relation between pressure, 
intermittency and the wake component of the mean velocity profile. Note that the approximate agreement of the stresses in figure $6(b)$ relies on our identification of $\delta_{99}$ with the channel half-width. It was in fact one of the reasons that led us to that identification. On the other hand, the discrepancies in the other two figures are relatively independent of the coordinate scaling

The overall picture is one in which pressure fluctuations in the boundary layer are less effective than the Reynolds stresses in homogenizing the velocity in the intermittent layer, leading to a higher mean velocity in that region. The steeper velocity gradient results in a larger overall energy production and dissipation in the boundary layer, and the resulting stronger vorticity creates stronger pressure Huctuations, which in turn lead to a faster redistribution of energy to the transverse velocity components.

\section{Spectra}

Before using the spatial spectra of the boundary layer to study the scales of the processes just discussed, the spectra have to be properly defined. The simulations were not rum long enough to compile meaningful frequency spectra of the largest scales, and it would have been impractical in any case to store enough information to compute them in more than a few isolated points. Moreover, it was shown by del Alamo \& Jiménez (2009) that the temporal and spatial spectra are not equivalent, and that substituting one for the other can lead to serious artefacts. A comparison between the experimental frequency spectra in boundary layers and the numerical wavenumber spectra in channels can be found in del Álamo \& Jiménez (2009) and in Hoyas \& Jiménez (2008). There is no problem in defining spatial spectra along the spanwise direction of the boundary layer, which is homogeneous, but the streamwise wavenumber spectrum does not strictly exist for spatially evolving flows. What is actually compiled in the simulations is the two-point correlation function of each spanwise Fourier mode, from where the two-dimensional $\left(k_{x}-k_{2}\right)$ spectra are computed as Fourier transforms. The details are given in the Appendix.

This requires symmetrizing the correlations, and implies an inhomogeneity error that can be estimated by computing the 'spectrum' of their antisymmetric parts. This was done for section BLS2 of the boundary layer, which is the only one whose correlations extend far enough within the uncontaminated simulation region to compute symmetrized spectra, and was used to estimate the longest useful spectral wavelength. The result, $\lambda_{x} \approx 165 \theta \approx 20 \delta_{99}$, corresponds to a turnover of the largest eddies, and is comparable to the lengths of the spectra available for the channels. Over that range, the antisymmetric 'spectrum' is at least an order of magnitude smaller than the symmetrized one, except for $E_{u k}$, which becomes more asymmetric for $\lambda_{x}>10 \delta_{99}$ and $y / \delta_{99}>0.8$. The spanwise width of the boundary-layer simulation is given in table 2, and is also comparable to those of the channels. The correlations of the other two boundary-layer sections, for which either the upstream or downstream leg falls outside the useful simulation range, have been symmetrized by copying their 'good' legs into their 'bad' ones.

In this section we mostly compare spectra from section BLS2 of the boundary layer, whose Reynolds number is $\delta^{+}=547$, with those of the C550 channel, for which $\delta^{+}=578$. Their intensity profiles are reproduced in figure $7(a)$. It is seen that the three intensities are stronger in the boundary layer, although less so for the streamwise component, and that the maximum differences are around $y / \delta=0.3-0.5$, which is just below the lower end of the intermittent region. The comparison of the 

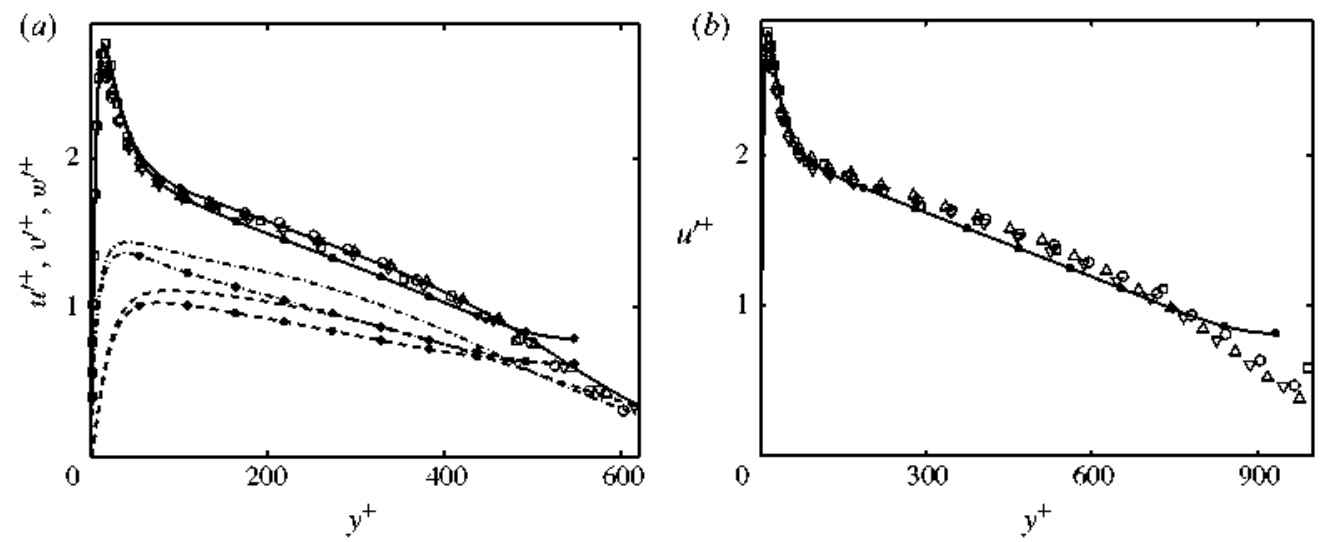

Figure 7. Intensities of the velocity fluctuations. (a) The lines with heavy dots are C550, and those without are BLS2. -,$u^{i+} ;-\cdots, v^{i+} ;-.-, w^{i+}$. Open symbols are from Erm \& Joubert (1991) and de Graafl \& Eaton (2000), at approximately the same $R e_{\tau}$, with the same notation as in figure 1. (b) Same for C 950.

pressure fluctuations is done in figure 2(a). The discrepancies in the two transverse components are consistent with the results of Hoyas \& Jiménez (2008), who found that they increase with the Reynolds number. The experiments surveyed in that paper were too noisy to identify any difference between the streamwise velocity intensities of the two kinds of flows, and the small differences observed in figure 7(a) for this velocity component, although interesting because they would remove the inconsistency that one velocity component should be different from the other two, require confirmation. Because of the particular interest of the streamwise component, the experimental results by Erm \& Joubert (1991) and de Graaff \& Eaton (2000) at similar Reynolds numbers are included in figure $7(a)$, where they agree with our simulation. Figure $7(b)$ displays the streamwise intensities at the Reynolds number of the C950 channel, compared with the available boundary-layer experiments at that Reynolds number, and also shows the slight excess of the boundary layers over the channel.

The Reynolds numbers of the present simulation are unfortunately too low to say much about the scaling of the locations for these differences. For example, none of the spectra has a linear range of length scales that could be used to define a logarithmic layer. Hoyas \& Jiménez (2008), who surveyed experimental data over a wider range of Reynolds numbers, concluded that the $v^{\prime}$ excess is centred around $y / \delta \approx 0.2$, and that of $w^{\prime}$ around $y / \delta \approx 0.4$. Buschmann et al. (2009) gave the same location for the $v^{t}$ discrepancy, but found that $w^{\prime}$ has an excess over a wider region $y / \delta \approx 0.2-0.5$. Both values are consistent with figure 7 , although simulations at higher Reynolds numbers are again required for confirmation.

The general structure of the spectra is shown in figure 8 , which contains streamwise and spanwise spectra of the two transverse velocity components and of the pressure. Over the range of Reynolds numbers of the three boundary-layer sections in table 2, the shortest and narrowest wavelengths of the spectra collapse reasonably well in wall units, while the widest and longest ones collapse better in outer units. Most of the spectra have ridges around $y / \delta=0.3-0.5$, with $\lambda_{z} \approx \delta$ and $\lambda_{x} \approx 2 \delta$, which agree approximately with earlier estimates of the scales of the transverse motions in channels (del Alamo \& Jiménez 2003; del Alamo et al. 2004). The spectra of the streamwise 

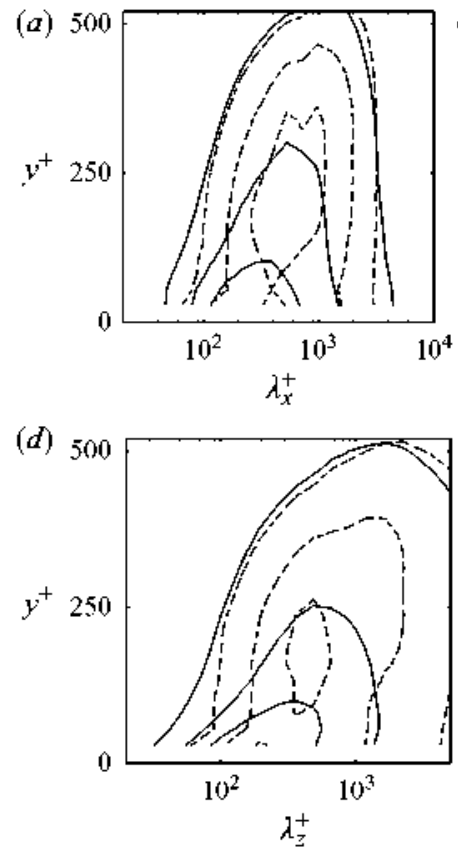

(b)

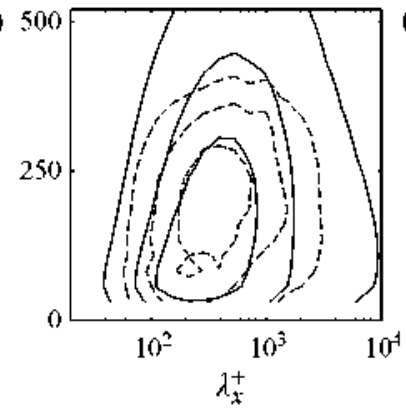

(e)

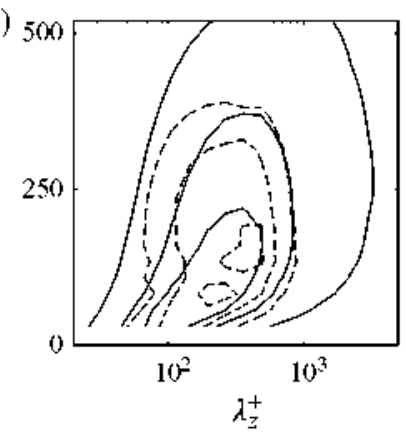

(c)

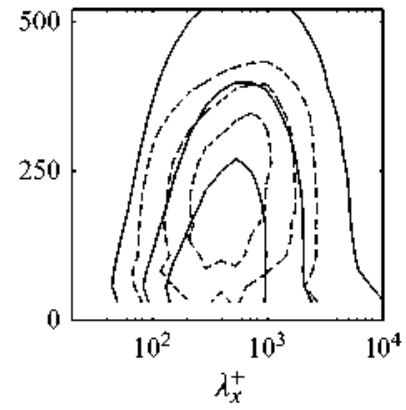

$(f)$

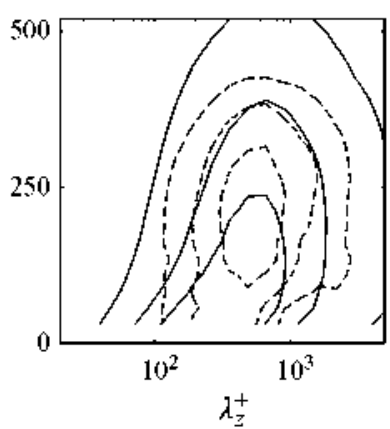

FIGURE 8. Pre-multiplied spectra as finnctions of $y$ and $\lambda_{x}$ in $(a-c)$, and of $\lambda_{z}$ in $(d-f)$, in wall scaling. The solid isolines are BLS2, and correspond to $(0.1,0.4,0.7)$ times the maximnm of each spectrum. The dashed isolines are the excess of the spectra of BLS2 over C550, and correspond to $(0.05,0.10,0.15)$ times the maximum of BLS2. $(a, d)$ Pressure $(b, e)$ Wall-normal velocity. (c, $f$ ) Spanwise velocity.

velocity are given in figure 10 , and will be discussed later. They are longer than both $p$ and $w$, but not wider. The wall-normal velocity is both shorter and narrower than the other two components, as first observed in the bufler layer by Kim et al. (1987). Together with the vertical correlation results in figure $5(b)$, these observations define the general geometry of the three velocity components. The structures of $u$ are long, those of $v$ are tall and those of $w$ are wide. The pressure fluctuations are as tall as the wall-normal velocity, but wider and somewhat longer. That is confirmed by visual inspection of the instantaneous flow fields both in the channel (not shown) and the boundary layer (figure 9), and, at least in the case of the velocities, is clearly connected with the effect of continuity. Even in isotropic turbulence, the root-meansquared longitudinal velocity derivatives, such as $\partial_{x} u$ or $\partial_{y} v$, are $\sqrt{2}$ times weaker than the transversal ones (Batchelor 1953).

Figure 8 also includes the excess in spectral energy of the boundary layer with respect to the channel, and it is sealed in inner units to minimize the differences in the buffer-layer length scales due to the slightly diflerent Reynolds numbers of the two flows. The differences away from the wall are not due to the Reynolds number, and survive both in wall and outer units. The boundary-layer spectra reach farther into the flow, and are always more intense than in the channel, with the main differences around $y / \delta=0.2-0.5$. That is consistent with the fluctuation profiles, but it is interesting that the largest differences are confined to wavelengths near the core of the respective spectra, with little evidence of changes in the width or length of the structures. 

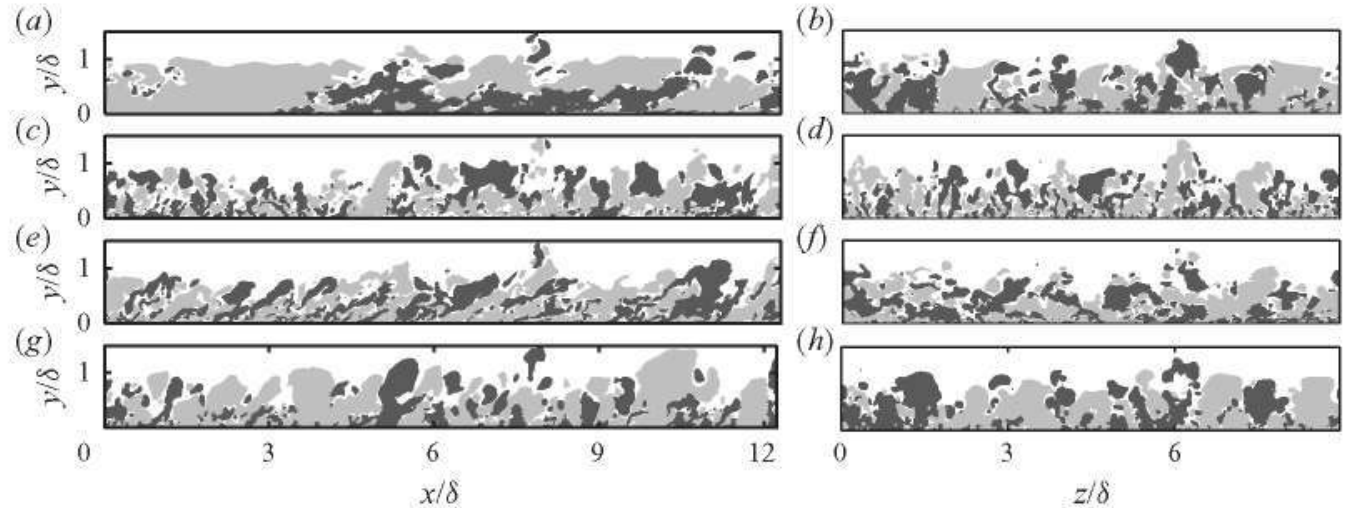

FIGURE 9. Instantaneous sections of the fluctuations in the boundary layer: $u(a, b), v(c, d)$, $w(e, f), p(g, h) .(a, c, e, g)$ The $x-y$ sections, in $R e_{\theta}=1670-2000$, and $(b, d, f, h)$ the $z-y$ sections at $\operatorname{Re}_{\theta}=1670$. All the fluctuations are normalized with the $x$-dependent friction velocity, and the coordinates are normalized with $\delta_{99}$ at $R e_{\theta}=1670$. In all the sections the dark areas are below -0.5 wall units, and the lighter ones above +0.5 .
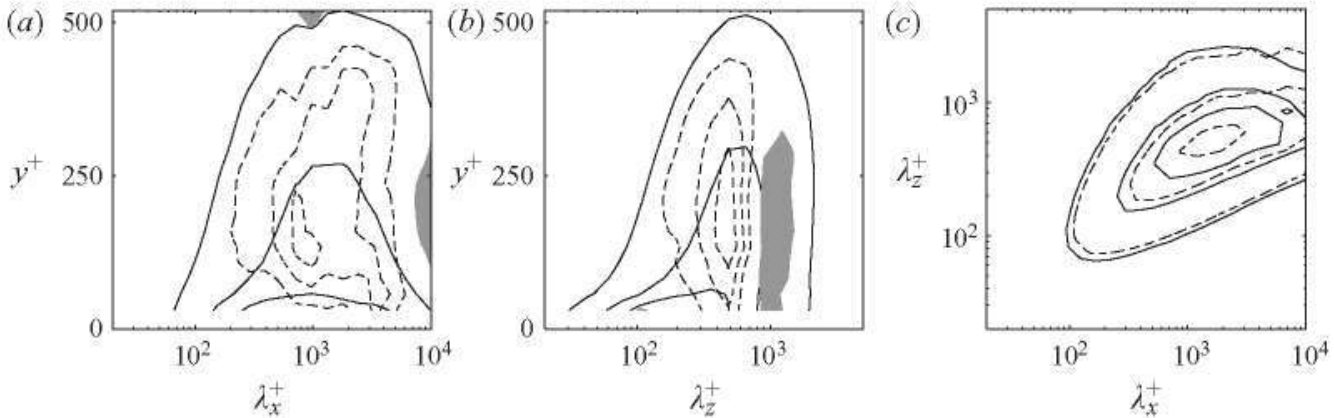

Figure 10. $(a-b)$ As in figure 8 , but for the streamwise velocity. The dashed isolines are $(0.04,0.08,0.12)$ times the maximum of BLS2. The shaded area is within the isoline $(-0.04)$. (c) Two-dimensional pre-multiplied spectrum of $u$, at $y / \delta=0.3 . \longrightarrow$, BLS2; ---, CH550. Spectra are normalized with $u_{\tau}^{2}$, and isolines are $(0.1,0.4,0.7)$ times the maximum of the boundary-layer spectrum.

The spectra of the streamwise velocity component are given in figures $10(a)$ and $10(b)$, and they look different from those of the transverse velocities. The shaded areas are negative, and show that the $u$ spectra of the boundary layer are not only slightly more intense that those of the channel, but that they are also displaced towards narrower wavelengths (Monty et al. 2007). In contrast, the only place where the spectra of the transverse velocities are less intense for the boundary layer than for the channel is above $y=0.9 \delta$, where the velocity fluctuations of the boundary layers decay into the free stream.

It turns out that this difference is an artefact of the one-dimensional representation. The two-dimensional spectra at $y / \delta=0.3$ are given in figure $10(c)$, and there is little evidence of a difference in spanwise scale. This is the height at which the two spectra differ most, but the only difference in the wave vector plane seems to be that the channel spectra are longer and less intense. The apparent difference in width is due to the energy missing in the long-wavelength end of the boundary layer, which is 
also the widest. Those long wavelengths are however the less reliable ones for the boundary layer. It was noted by Jiménez \& Hoyas (2008) that there were at the time essentially no experiments or simulations in which the very long streamwise scales of the $u$ component were clearly resolved, although the available evidence suggested $\lambda_{x} \lesssim 25 \delta$. This is also the limit of our boundary-layer spectra, and we have seen at the beginning of this section that the difficulty is not only formal; that is, the distance beyond which the boundary layer can no longer be considered homogeneous. Recent experiments by Monty et al. (2009) roughly confirm those conclusions. They show that the premultiplied temporal spectra of $u$ in pipes and channels have plateaus extending to $\lambda_{x} / \delta \leqslant 20$, while those of boundary layers peak at $\lambda_{x} \approx 3-6$. On the other hand, what figures 8 and $10(c)$ show is that, within the limits in which both flows can be considered as approximately parallel, the spatial scales of the boundary layer are essentially the same as those of the channel.

A similar explanation holds for the narrower spectra of $v$ in figure $8(e)$, with respect to the other velocity components or to the pressure. The wall-normal velocity has a much shorter spectrum than any of the other components, lacking inactive motions (del Álamo et al. 2004; Hoyas \& Jiménez 2006). Its width is similar to that of either $u$ or $p$ at those short wavelengths, but the integrated spanwise spectra for any of those fluctuations are broadened by their wider components at longer wavelengths, which are missing for $v$.

Since the amplitudes of the fluctuations are different in both flows, and since we have shown that this diflerence is due to the different structure of the Reynolds stresses in the intermittent layer above that region, it appears that the wavelength-selection mechanism of wall-bounded shear hows is relatively independent of the amplitude, and that the two reside in diflerent parts of the How. While the size of structures is controlled by the region around $y / 8 \approx 0.3-0.5$, the amplitude is, at least in part, influenced by processes in the outer layers, where intermittency matters, and where dissipation dominates over production.

\subsection{The geometry of the vortical structures}

To get some idea of the geometry of the structures represented by the spectra just described, figure 11 displays an isosurface of the discriminant of the velocity gradient tensor from the present simulation (Chong et al. 1998). As in del Álamo et al. (2006), the discriminant is thresholded with a constant fraction of its standard deviation, although, to compensate for the streamwise inhomogeneity of the boundary layer, the discriminant in local wall units is thresholded with its standard deviation, compiled as a single function of $y / \delta$. The thresholding factor $\left(2 \times 10^{-3}\right)$ was chosen to retain a volume fraction compatible with visual interpretation (figure 12a), with the result that figure 11 spans mostly the intermittent region. The vortices become too dense farther into the boundary layer to appreciate their arrangement.

Figure 11 $(a)$ is a vertical view of the discriminant, looking into the plane of the wall. Figure $11(b)$ is a perspective view of the same isosurface, to aid in the interpretation. Both reveal a multitude of vortices and arches. However, although there is no doubt that many of them can be described as hairpins, and although we found at least one clear instance of a 'train' of three aligned hairpins (Adrian 2007), most of them are oriented randomly, rather than with the predominant shear. It is difficult, at least for us, to describe the structure of these figures as an ordered hairpin 'forest'. This disagrees with recent similar representations by Wu \& Moin (2009), but was probably to be expected. The Reynolds number of the present simulation is at least twice that of Wu \& Moin (2009), and the ratio between the vortex intensity and the mean velocity 
(a)

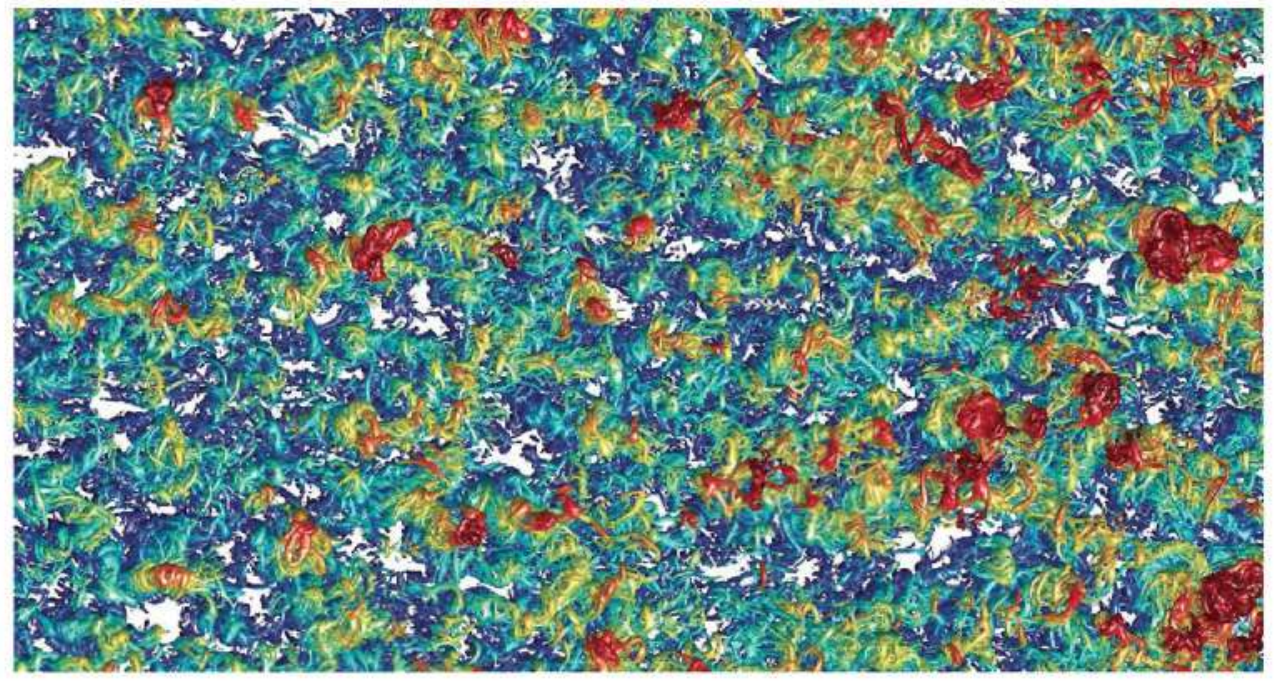

(b)

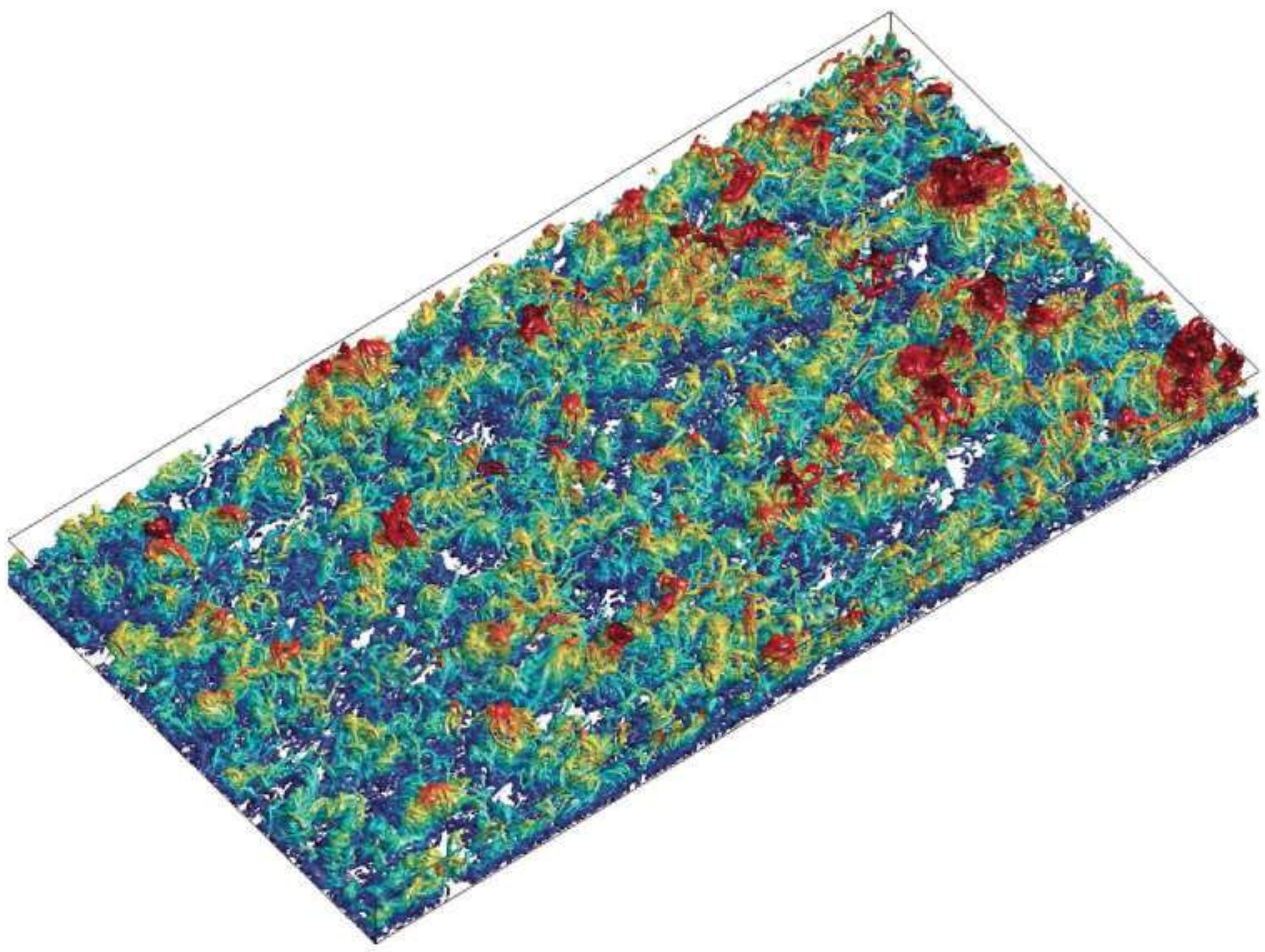

Figure 11. Isosurface of the discriminant of the velocity gradient tensor of the present simulation. (a) Top view. (b) Perspective view. In both cases the flow is from left to right, and the wall-parallel dimensions of the box are approximately $18 \times 9$ times the boundary-layer thickness at the centre of the box, spanning $R e_{\theta} \approx 1420-1900$. The isosurface is coloured by the distance to the wall, from $y / \delta \approx 0.3-0.4$ for the deepest blue, to $y \approx \delta$ for the brightest red.

gradient is also higher. If we assume that the dissipation is roughly in equilibrium with the energy production, $v \omega^{\prime 2} \approx \tau \partial_{y} U$, we obtain for the mean enstrophy,

$$
\omega^{\prime} / \partial_{y} U \approx\left(\tau^{+} / \partial_{y} U^{+}\right)^{1 / 2},
$$



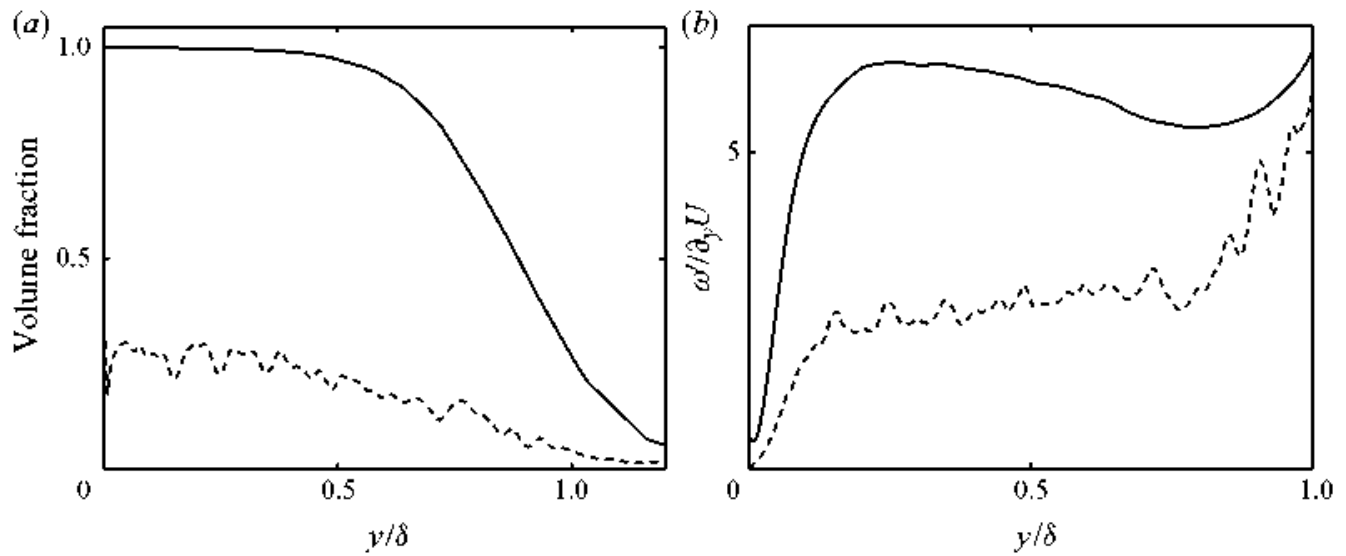

FIGURE 12. $(a)$ The dashed line is the volume fraction bounded by the isosurfaces in fignre 11 . The solid line is the intermittency factor. (b) The solid line is the mean vorticity magnitude, divided by local mean velocity gradient. The dashed line is the same ratio for the discriminant threshold nsed in fignre $11,2 D^{1 / 6} / 3$, as explained in the text.

which in the logarithmic layer is

$$
\omega^{\prime} / \partial_{y} U \approx\left(\kappa y^{+}\right)^{1 / 2},
$$

where $k$ is the Kármán constant. This ratio increases with the Reynolds number, and figure 12(b) shows that it is approximately equal to six in our simulation. The discriminant in figure 11 is a sixth power of the velocity gradients, and cannot be directly compared with the enstrophy, but in our simulation $D^{\prime 1 / 6} \approx 1.5 \omega^{\prime}$. The threshold used in figure 11 has therefore been included in figure $12(b)$ as $2 D^{1 / 6} / 3$, for comparison.

Since the typical maximum vorticity of the compact vortices is a few times $\omega^{\prime}$ (Jiménez et al. 1993; Tanahashi et al. 2004), they are essentially decoupled from the mean velocity profile, and are approximately isotropic. In fact, the intensities of the three vorticity components are roughly equal above $y^{+} \approx 50$, and their spectra also approximately agree with each other above that level. It was shown by Tanahashi et al. (2004) that the properties of the individual vortices in a turbulent channel are essentially identical to those in isotropic turbulence at similar Reynolds numbers, and the same seems to be the case in the boundary layer. The vortices in figure 11 $(a)$ resemble much more the 'tangles' described by del Alamo et al. (2006) and Flores, Jiménez \& del Álamo (2007), than the ordered arrays in Wu \& Moin (2009), and it is especially interesting that the tallest vortical regions, which could be considered as the 'leading edges' of the diffusion of the turbulent region into the free stream, resemble much more isotropic ejections than organized hairpins.

The low Reynolds number of the simulation of Wu \& Moin (2009) suggests that its perceived order may be a transitional effect. Its friction coefficients are included in figure $1(a)$, and fall within the transition-dominated region, where the eddies have travelled less than one turnover from their initial formation, and retain some of the properties with which they where created. Figure 11 is drawn for Reynolds numbers beyond $R e_{\theta}=1400$ and has presumably lost any transitional order it may have had. Our simulation bypasses transition, and would therefore probably be everywhere diflerent from Wu \& Moin (2009), but the newer one by Schlatter et al. (2009) goes through natural transition and, while its initial region is broadly similar to Wu \& 
Moin (2009), it becomes as disorganized as figure 11 at comparable Reynolds numbers (P. Schlatter, personal communication 2009).

On the other hand, it is plausible that, even if the small-scale vortices are disorganized, some organization could be recovered for the larger-scale eddies, in the same way as a self-similar geometry was recovered for the vortex clusters in del Álamo et al. (2006) and Flores et al. (2007). It is already clear from figure 11 that the vortices are arranged in large streamwise streaks, about one boundarylayer thickness wide, knotting into ejections every few boundary-layer thicknesses, in agreement with the spectra of the velocities. An attempt to highlight any further structure was made by redrawing figure 11 using the discriminant of a velocity field that had been filtered with a Gaussian window with semiaxes $150 \times 40 \times 100$ wall units in the three coordinate directions. The resulting figures are somewhat cleaner, lacking many of the thinnest vortices in figure 11, but their overall structure is visually almost indistinguishable from the figures printed above, and they are therefore not shown. It nevertheless remains possible that much coarser filtering would result in the clearer emergence of large structures closer to those observed in transition, but such filters are difficult to implement at the limited Reynolds numbers of the existing simulations.

\section{Conclusions}

We have used the comparison of older simulations of turbulent channels with a new simulation of the zero-pressure-gradient boundary layer at moderate Reynolds number $\left(\delta^{+}=400-700\right)$, to study the effects of the outer intermittent region of the boundary layer on the structure of the large scales of the flow. The domain of the new simulation is long enough for the effect of the inflow conditions to be forgotten, and agrees with experimental observations for boundary layers beyond the point at which the eflect of the initial trip becomes negligible. This, however, requires discarding the initial $25 \%$ of the simulation domain, equivalent to about two eddy turnovers, and limits the minimum useful Reynolds number to $R e_{\theta} \approx 1100$.

In agreement with previous observations, it is found that the fluctuations of the transverse velocity components, and of the pressure, are stronger in the boundary layer than in the channel, and this is traced to the reduced effectiveness of the Reynolds stresses in the potential parts of the flow in the intermittent region. This leads to a higher mean velocity in this part of the flow, and to the well-known stronger wake component of the mean velocity profile in the boundary layer.

The task of homogenizing the velocities of the potential and rotational regions is taken over by the pressure, whose fluctuations are stronger than in the channel due to the large differences in the mean enstrophy of the two types of fluid. The stronger pressure fluctuations modify the flow globally, and are the origin of the stronger transverse velocity Huctuations in the boundary layer. Although our group, among others, had previously concluded that the streamwise velocity fluctuations are similar for internal and external flows (Jiménez \& Hoyas 2008), the present results suggest that this is probably not so, although the differences are smaller than for the other components.

Within the range of streamwise distances in which the boundary layer can be approximately be considered as a parallel flow $(\Delta x \approx \pm 20 \delta$, corresponding to $\Delta \delta \approx$ $\pm 0.3 \delta$ ), there is essentially no difference between the wall-parallel scales of the velocity fluctuations of the two flows. In both cases it is found that the streamwise velocity fluctuations are long, those of the spanwise velocity are wide, and those 
of the wall-normal component are tall. The pressure fluctuations are as tall as the wall-normal velocity, but wider and somewhat shorter. The quadrant analysis of the Reynolds stresses clearly indicates that the large-scale ejections from one side of the channel cross deeply into the other half. This is confirmed by the velocity correlations of the large-scale velocities, which are consistent with structures whose wall-normal velocities tend to be symmetric with respect to the channel centreline, but whose streamwise velocities are antisymmetric.

All those effects are absent from the boundary layer, but they are substituted by the pressure fluctuations, which provide an alternative mechanism to accelerate the How. Although turbulent ejections are clearly still present in the intermittent layer, they do not constitute the primary mechanism for momentum transfer, and the mean tangential Reynolds stress of the rotational fluid in that region is strongly 'countergradient'. A visualization of the vortices in the boundary layer does not support, in our interpretation, a model in terms of a moderately ordered hairpin forest.

The largest differences between the boundary layers and channels are located just below the intermittent region, and just above the logarithmic layer, $(y / \delta=0.3-0.5)$. Together with the similarity of the scales of the two types of flows, this suggests that the wavelength selection mechanism for the largest scales of wall-bounded flows resides in that region. The limited Reynolds numbers of the present simulation prevent us from confirming or denying the conclusion in Jiménez \& Hoyas (2008) and Buschmann et al. (2009) that this location scales in outer units, but the mechanism outlined above, by which the pressure fluctuations in the intermittent layer are responsible for the diflerences between the two Hows, together with the outer scaling of the intermittency factor, would support that conclusion. In addition, the same mechanism suggests that the conjecture by Buschmann et al. (2009) that two diflerent eflects might be responsible for the $v$ and $w$ structures is probably unnecessary.

This work was supported in part by grants TRA2006-08226 and TRA2009-11498 of the Spanish CICYT, and by the EU FP6 Wallturb Strep AST4-CT-2005-516008. The computations were made possible by generous grants of computer time from the Barcelona Supercomputing Centre, and by the equally generous collaboration of the Port d'Informació Cientifica (PIC), which lent their mass storage facilities to archive raw data. M.P.S. was supported in part by the EU FP5 Training and Mobility Network HPRN-CT-2002-00300, and S.H. and Y.M. by the Spanish Ministry of Education and Science, under the Juan de la Cierva programme.

\section{Appendix. The estimation of the streamwise spectra}

What is actually compiled in the simulation is the two-point correlation function of each spanwise Fourier mode, which, for two arbitrary variables ' $a$ ' and ' $b$ ', is defined as

$$
C_{a b}\left(x, r, k_{z}\right)=\left\langle\widehat{a}\left(x, k_{z}\right) \hat{b}^{*}\left(x+r, k_{z}\right)\right\rangle,
$$

where $\widehat{a}$ is the spanwise Fourier component of ( $a$ ) corresponding to the wavenumber $k_{z}$, and \langle\rangle denotes averaging over time. Note that (A 1) is statistically real because of the reflection symmetry between $k_{z}$ and $-k_{z}$, even if the Fourier components are complex. When the flow is homogeneous along the streamwise direction, $C_{a b}$ is only a function of $r$ and, if $a=b$, the autocorrelation $C_{a a}$ is symmetric with respect to $r=0$. In that case the energy spectrum can be defined as the Fourier transform 
(Hinze 1975)

$$
E_{a t a}\left(k_{x}, k_{z}\right)=\pi^{-1} \int_{-\infty}^{\infty} C_{a t u}\left(r, k_{z}\right) \exp \left(\mathrm{i} k_{x} r\right) \mathrm{d} r=2 \pi^{-1} \int_{0}^{\infty} C_{a a}\left(r, k_{z}\right) \cos \left(k_{x} r\right) \mathrm{d} r
$$

where the last expression uses the symmetry of $C_{a a}(r)$. If, as in the case of the spatially growing layer, the correlation is not symmetric, the spectrum defined by the first equation in (A 2) has an imaginary part that can be used as a measure of the error resulting from assuming the flow homogeneous. In addition, in the practical case in which the sampling interval is finite, the integrals in (A2) have to be windowed to avoid the implied discontinuity at the interval boundaries. We therefore derive the spectrum at a point $x$ from the symmetrized correlation.

$$
E_{a t a}\left(k_{x}, k_{z}\right)=\pi^{-1} \int_{0}^{L}\left[C_{a t u}\left(r, k_{z}\right)+C_{u t a}\left(-r, k_{z}\right)\right] \cos \left(k_{x} r\right) W(r / L) \mathrm{d} r
$$

where $x \pm L$ is the sampling interval, and

$$
W(\xi)=0.35875-0.48829 \cos (\pi \xi)+0.14128 \cos (2 \pi \xi)-0.01168 \cos (3 \pi \xi),
$$

is a Blackman-Harris smoothing window (Harris 1978). Although the longest finite wavelength in this spectrum is $\lambda_{x}=2 L$, the effect of windowing is to smooth the spectrum over approximately three neighbouring wavenumbers, and to damp wavelengths longer than approximately $L$. Therefore, after using (A 3 ), we resample the spectra to the interval $k_{x} \geqslant 2 \pi / L$ by accumulating every two neighbouring streamwise wavenumbers. The inhomogeneity error is estimated by computing the antisymmetric contribution

$$
F_{a a}\left(k_{x}, k_{z}\right)=\pi^{-1} \int_{0}^{L}\left[C_{a a}\left(r, k_{z}\right)-C_{a t z}\left(-r, k_{z}\right)\right] \sin \left(k_{x} r\right) W(r / L) \mathrm{d} r .
$$

The $u-v$ correlation is not symmetric with respect to $r=0$, even in homogeneous flows, but (A 3) can still be used to define a real cospectrum that retains the property that its integral is the $\langle a v\rangle$ Reynolds stress.

Because the statistical averaging can only be done over time, instead of over $x$ and time, as in homogeneous Hows, the spectra computed in this way are noisier than in channels. To remedy this, the correlations are first averaged over 101 neighbouring $x$ points, equivalent to about one boundary-layer thickness, and the spectra are smoothed for display by aggregating them into wavenumber bands over both $k_{x}$ and $k_{3}$, whose widths $n_{j}$ in terms of discrete wave indices are determined from a Fibonacci-like sequence

$$
n_{j}=n_{j-1}+n_{j-5}
$$

to avoid interpolating over non-integers. The widths of the first few bands are $n_{j}=1, j=1 \ldots 5$, so that the first few spectral modes are not modified, but they soon settle into an exponential sequence $n_{j} \approx 1.325 n_{j-1}$. If the wavenumber with index $m$ is $k_{m}=2 \pi m / L$, the index associated with the band between $m_{1}$ and $m_{2}$ is defined as $\sqrt{m_{1} m_{2}}$. To maintain consistency, the same aggregation procedure is used for the spectra of the boundary layer and of the channels.

\section{REFERENCES}

AdrLan, R. J. 2007 Hairpin vortex organization in wall turbulence. Phys. Fluids 19, 041301.

Alam, M. \& SANDHAM, N. D. 2000 Direct numerical simulation of short laminar separation bubbles with turbuleut reattachmeut. J. Fluid Mech. 410, 1-28. 
Del Álamo. J. C. \& Jiménez, J. 2003 Spectra of very large anisotropic scales in turbuleut chaunels. Phys. Fluids 15, L41-L44.

DEl Álamo, J. C. \& JimÉnez, J. 2009 Estimation of turbulent convection velocities and corrections to Taylor's approximation. J. Fluid Mech. 640, 5-26.

del Álamo. J. C, Jiménez. J., Zandonade. P. \& Moser. R. D. 2004 Scaling of the energy spectra of turbulent channels. J. Fluid Mech. 500, 135-144.

DEl Álamo, J. C., JimÉnez, J., Zandonade, P. \& Moser, R. D. 2006 Self-similar vortex clusters in the logarithmic region. J. Fluid Mech. 561, 329-358.

AntoniA, R. A., Teittel, M., KiM. J. \& Browne, L. W. B. 1992 Low-Reynolds-number effects in a fully developed turbulent chamel flow. J. Fhid Mech. 236, 579-605.

BATCHELOR, G. K. 1953 The Theory of Homogeneous Turbulence, pp. 45-47. Cambridge University Press.

Bisset, D. K., Hunt, J. C. R. \& Rogers. M. M. 2002 The turbulent/non-turbulent interface bounding a far wake. J. Fluid Mech. 451, 383-410.

Buschmann, M. H., Kempe, T., Indinger. T. \& Gad-el-Hak. M. 2009 Normal and crossflow Reynolds stresses differences between confined and semi-conhined flows. In Turbuletce, Heat and Mass Transfer 6 (ed. K. Hanjalić, Y. Nagano \& S. Jakirlić), pp. 1-7. Begell House.

Chong, M. S., Sorla, J., Perry, A. E., Chacin, J. Cantwell, B. J. \& Na, Y. 1998 Turbulent structures of wall-bounded shear flows found using DNS data. J. Fluid Mech. 357, 225247.

Corrsin. S. \& Kistler, A. L. 1955 Free-stream boundaries of turbulent flows. Tech. Rep. 1244. NACA.

ERm. L. P. \& Joubert, P. N. 1991 Low-Reynolds-number turbulent boundary layers. J. Fluid Mech. 230, $1-44$.

Farabee, T. M. \& Casarella. M. J. 1991 Spectral features of wall pressure fluctuations beneath turbulent boundary layers. Phys. Fluids A 3, 2410-2420.

Ferrante, A. \& Elghobashi, S. 2005 Reynolds number effect on drag reduction in a microbubbleladen spatially developing turbulent boundary layer. J. Flitid Mech. 543, 93-106.

Fiedler, H. E. \& HEAD, M. R. 1966 Intermittency measurements in the turbulent boundary layer. J. Fluid Mech. 25, 719-735.

FloRes, O.. Jiménez, J. \& DEL Álamo, J. C. 2007 Vorticity organization in the onter layer of turbulent channels with disturbed walls. J. Fluid Mech. 591, 145-154.

DE GraAf, D. B. \& EaTon, J. K. 2000 Reynolds number scaling of the flat-plate turbulent boundary layer. J. Flitid Mech. 422, 319-346.

HARRIS, F. J. 1978 On the use of windows for harmonic analysis with the discrete Fourier transform. Proc. IEEE 66, 51-83.

Hedley, T. B. \& Keffer, J. F. 1974 Some turbulent/non-turbulent properties of the outer intermittent region of a boundary layer. J. Fluid Mech. 64, 645-678.

Hinze, J. 1975 Turbulence, 2nd edn, pp. 61-68. McGraw-Hill.

HoYas, S. \& JimÉnez, J. 2006 Scaling of the velocity fluctuations in turbulent channels up to $R e_{i}=2003$. Phys. Fluids 18, 011702 .

HoYAs, S. \& JimÉNEZ, J. 2008 Reynolds number eflects on the Reynolds-stress budgets in turbulent channels. Phys. Fhids 20, 101511.

Hu, Z. Morley, C. \& Sandham, N. 2006 Wall pressure and shear stress spectra from direct simulations of chauuel flow. $A I A A$ J. 44, 1541-1549.

JimÉNEZ, J. \& Hoyas, S. 2008 Turbulent fluctuations above the buffer layer of wall-bounded flows. J. Fluid Mech. 611, 215-236.

Jiménez. J., Hoyas, S., Simens. M. P. \& Mizuno. Y. 2009 Comparisou of turbulent bouudary layers and channels from direct numerical simulation. In Sixth International Symposium on Turbulence and Shear Flow Phenomena (ed. H. J. Sung \& H. Choi), Pp. 289294.

JimÉnEz, J., WRAY, A. A., SAFfman, P. G. \& RoGallo, R. S. 1993 The structure of intense vorticity in isotropic turbulence. J. Fluid Mech 255, 65-90.

KhujaDze, G. \& OberLACK, M. 2004 DNS and scaling laws from new symmetry groups of ZPG turbulent boundary layer flow. Theor. Comput. Fhid Dyn. 18, 391-441. 
KIM, J. 1989 On the strncture of pressure Hnctuations in simulated turbulent channel How. J. Fluid Mech. 239, 157-194.

KIM, J. \& MoIN, P. 1985 Application of a fractional-step method to incompressible Navier-Stokes equations. J. Conıut. Phys. 59, 308-323.

Kim, J., Mon, P. \& Moser, R. D. 1987 Turbulence statistics in fnlly developed channel how at low Reynolds nnmber. J. Fluid Mech. 177, 133-166.

Kovasznay, L., Kibens, V. \& BlackWElder, R. 1970 Large-scale motion in the intermittent region of a turbulent boundary layer. J. Fhid Mech. 41, 283-325.

LeE. S.-H. \& Sung. H. J. 2007 Direct numerical simulation of the turbuleut boundary layer over a rod-roughened wall. J. Fluid Mech. 584, 125-146.

Lund, T. S., Wu, X. \& Souires, K. D. 1998 Generation of turbulent inflow data for spatiallydeveloping boundary layer simulations. J. Comput. Phvs. 140, 233-258.

Monty, J. P., Hutchins, N., NG, H. C. H., Marusic, I. \& Chong., M. S. 2009 A comparison of turbulent pipe, channel and boundary layer fows. J. Fluid Mech. 632, 431-442.

Monty, J. P., Stewart, J. A., Williams. R. C. \& Chong., M. S. 2007 Large-scale features in turbulent pipe and channel flows. J. Fluid Mech. 589, 147-156.

Murlis, J.. Tsai, H. \& Bradshaw, P. 1982 The structure of turbulent boundary layers at low Reynolds numbers. J. Fluid Mech. 122, 13-56.

Nagara jan, S., Lele, S. K. \& Ferziger, J. H. 2003 A robust high-order compact method for large eddy simulation. J. Comput. Phys. 191, 329-419.

Nakagawa. H. \& NeZU. I. 1977 Prediction of the contributions to the Reynolds stress from bursting events in open channel How. J. Fluid Mech. 80, 99-128.

Nikirin, N. 2007 Spatial periodicity of spatially evolving turbulent flow caused by inflow boundary condition. Phys. Fluids 19, 091703.

Perot, J. B. 1993 An analysis of the fractional step method. J. Comput. Phys. 108, 51-58.

Pope, S. 2000 Turbulent Flows. Cambridge University Press, pp. 506-515.

SCHEwe, G. 1983 On the structure and resolution of wall pressure fluctuations associated with turbulent boundary layer flow. J. Fhid Mech. 134, 311-328.

Schlatter, P. Örlü, R., Li, Q., Fransson, J., Johansson, A., Alfredsson, P. H. \& Henningson, D. S. 2009 Turbulent boundary layers up to $R e_{e}=2500$ through simulation and experiments. Pliys. Fluids 21, 05702 .

Simens. M. P. 2008 The study and control of wall-bounded flows. PhD thesis, Aeronautics, Universidad Politécnica, Madrid, Spain, http://oa.upm.es/1047/.

Simens, M. P., JimÉnez, J., HoYAs, S. \& Mizuno, Y. 2009 A high-resolution code for turbulent boundary layers. J. Comput. Phys. 228, 4218-4231.

Skote, M. Haritonides, J. \& Henningson, D. 2002 Varicose instabilities in turbulent boundary layers. Phys. Fluids 14, 2309-2323.

Skote. M. \& Henningson. D. S. 2002 Direct numerical simulation of a separated turbulent boundary layer. J. Fluid Mech. 471, 107-136.

SPAlART, P. R. 1988 Direct simulation of a turbulent boundary layer up to $R e_{\theta}=1410$. J. Fluid Mech. 187, 61-98.

SPalart, P. R. COLEMan, G. N. \& Johnstone, R. 2009 Retraction: direct numerical simulation of the Ekman layer: a step in Reynolds number, and cautious support for a log law with a shifted origin. Phys. Fluids 21, 109901.

Spalart, P. R. Moser, R. D. \& Rogers, M. M. 1991 Spectral method for the NavierStokes equations with one inhnite and two periodic dimensions. J. Comput. Phys. 96, 297324.

SPALART, P. R. \& WATMUfF, J. H. 1993 Experimental and numerical study of a turbulent boundary layer with pressure gradients. J. Fluid Mech. 249, 337-371.

Tanahashi. M., Kang. S.-J.. Miyamoto, T., Shiokawa. S. \& Miyauchi, T. 2004 Scaling law of hine scale eddies in turbulent channel How at to $R e_{\mathrm{\tau}}=800$. Int J. Heat Fluid Flow' 25, 331340.

Tsuji, Y.. Fransson. J. H. M., Alfredsson, P. H. \& Johansson, A. V. 2007 Pressure statistics aud their scaling in high-Reynolds-number turbulent boundary layers. $J$. Fluid Mech. 585, 1-40.

Wallace. J. M., Eckelmann, H. \& Brodkey. R. S. 1972 The wall region in turbulent shear flow. J. Fluid Mech. 64, 39-48. 
Westerwel, J., Fukushima, C., Pedersen, J. M. \& Hunt, J. C. R. 2009 Momentnm and scalar transport at the turbulent/non-turbulent interface of a jet. J. Fluid Mech. 631, 199230.

Wu. X. \& Mon, P. 2009 Direct numerical simulation of turbulence in a nominally zero-pressuregradient flat-plate bonndary layer. J. Fluid Mech. 630, 5-41.

Wygnanski, I. J. \& Fiedler, H. E. 1970 The two-dimensional mixing region. J. Flinid Mech. 41, $327-361$. 